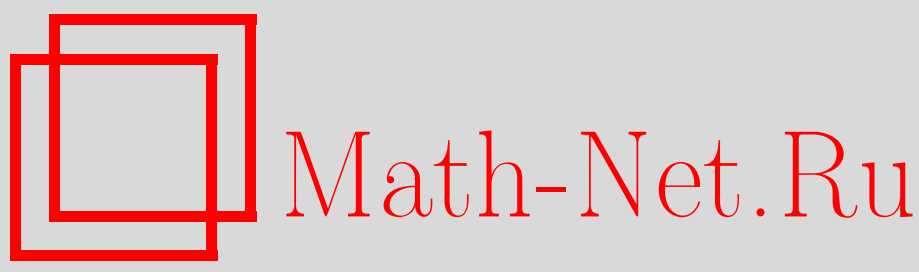

Е. А. Севастьянов, О проблеме Хаара для знакочувствительных аппроксимаций, Матем. сб., 1997, том 188, номер 2, 95-128

DOI: https://doi.org/10.4213/sm203

Использование Общероссийского математического портала Math-Net.Ru подразумевает, что вы прочитали и согласны с пользовательским соглашением http://www . mathnet.ru/rus/agreement

Параметры загрузки:

IP : 54.92 .164 .108

26 апреля 2023 г., 16:07:37 
УДК 517.51

\author{
Е. А. Севастьянов
}

\title{
О проблеме Хаара для знакочувствительных аппроксимаций
}

Проблема Хаара для знакочувствительных аппроксимаций заключается в отыскании необходимых и достаточных условий на знакочувствительный вес $p(x)=\left(p_{-}(x), p_{+}(x)\right), x \in E \subset \mathbb{R}$, и конечномерное подпространство $L$ пространства $C(E)$ непрерьвных на компакте $E$ функций $f$, при которых для любой $f \in C(E)$ в $L$ существует единственньй элемент наилучшего приближения со знакочувствительным весом $p$. Ряд таких условий устанавливается в данной статье. Выясняется, что эти условия существенньм образом связаны с топологическими свойствами аннуляторов функций $p_{-}(x), p_{+}(x)$, в частности, дается описание знакочувствительных весов $p=\left(p_{-}, p_{+}\right)$, для которых проблема Хаара имеет решение в форме условия, найденного Хааром для равномерных аппроксимаций, т.е. для случая $p(x) \equiv(1,1)$.

Библиография: 18 названий.

\section{Введение}

Пусть на некотором метрическом компакте $E$ задана линейно независимая система непрерывных действительнозначных функций $\varphi_{1}(x), \ldots, \varphi_{n}(x), x \in E$, card $E \geqslant n$. Рассмотрим равномерные приближения непрерывных на $E$ функций $f$ элементами $l=c_{1} \varphi_{1}+\cdots+c_{n} \varphi_{n}$ линейного подпространства $L$ с базисом $\varphi_{1}, \ldots, \varphi_{n}$. Элементом (полиномом) наилучшего равномерного приближсения непрерывной на $E$ функции $f$ назьвается такой элемент $l^{*} \in L$, что $\left\|l^{*}-f\right\|_{E}=\inf \left\{\|l-f\|_{E}: l \in L\right\}$, где $\|\cdot\|_{E}-$ равномерная норма на $E$. Сушествование элемента $l^{*}$ гарантируется для системы $\left\{1, x, \ldots, x^{n-1}\right\}$ теоремой Э. Бореля [1], для произвольной линейной независимой системы - известным обобщением этой теоремы (см., например, [2, с. 17]).

Проблема Хаара в классической постановке заключается в отыскании необходимых и достаточных условий, которьм должна удовлетворять система $\left\{\varphi_{1}, \ldots, \varphi_{n}\right\}$ (подпространство $L$ ), чтобы для каждой непрерывной на $E$ функции $f$ ее элемент наилучшего равномерного приближения был единственньм. Эта проблема была поставлена и решена А. Хааром в работе [3]: для того чтобы каждая непрерывная на $E$ функция $f$ имела в $L$ единственный элемент наилучшего приближения необходимо и достаточно, чтобы каждый полином $c_{1} \varphi_{1}(x)+\cdots+c_{n} \varphi_{n}(x)$ имел на $E$ не более $n-1$ различных нулей. Последнее условие получило название "условие Хаара" (см., например, [4, с. 93]).

Для равномерных аппроксимаций с произвольным полунепрерывным сверху знакочувствительным весом $p(x)=\left(p_{-}(x), p_{+}(x)\right)$ (определение этого понятия см. ниже) проблема Хаара (точнее, соответствующее обобщение ее классической постановки) заключается уже в отыскании необходимых и достаточных условий, 
которьм должна удовлетворять система (знакочувствительньй вес $p$, конечномерное подпространство $L$ ), чтобы для каждой непрерывной на компакте $E$ функции $f$ ее элемент $l(p, L, f)$ наилучшего приближения существовал и был единственньм (определение $l(p, L, f)$ дано ниже).

Настоящая работа посвящена решению этой задачи. В $\S 1$ устанавливается общий критерий единственности, в $\S 2$ - теоремы о так называемом чебьшёвском продолжении системы функций, в $\S 3$ результаты $\S \S 1,2$ используются для получения различных аналогов и обобшений классической теоремы Хаара. При этом значительное внимание в работе уделяется возможно более полному описанию весов $p$, для которых справедливы обобщения теоремы Хаара, приближающиеся по форме к оригинальной формулировке. В связи с этим отметим, что, видимо, первый результат в этом направлении содержится в книге М. Г. Крейна и А. А. Нудельмана [5], в которой на с. 491 отмечается, что теорема Хаара сохраняется в пространстве $B\left(\varphi_{+}, \varphi_{-}\right)$с несимметричной нормой, что фактически соответствует равномерным аппроксимациям со знакочувствительным весом $p=\left(p_{-}, p_{+}\right)$, функции $p_{-}(x)$ и $p_{+}(x)$ непрерывны и строго положительны на отрезке $E=[a, b]$. Доказательство этого факта легко получить соответствуюшими изменениями некоторых деталей доказательства самой теоремы Хаара.Принципиальные трудности при обобщении теоремы Хаара возникают в случае, когда неотрицательные компоненты $p_{-}(x)$ и $p_{+}(x)$ знакочувствительного веса $p(x)$, будучи произвольньми полунепрерывными сверху функциями, независимо друг от друга обращаются в нуль на тех или иных подмножествах компакта $E$.

Исходные обозначения и определения (см. [6]). Пусть $a \in \mathbb{R}, a^{+}=$ $\max \{a, 0\}, a^{-}=(-a)^{+}, a=a^{+}-a^{-},|a|=a^{+}+a^{-}$. Упорядоченную пару $p(x)=\left(p_{-}(x), p_{+}(x)\right)$ неотрицательных (принимающих и значение $+\infty$ ) функций $p_{-}$и $p_{+}$, определенных на некотором множестве $E$, назовем знакочувствительным весом на $E$; $p$ назовем ограниченным, полунепрерывным сверху и т.п., если таковы $p_{-}$и $p_{+} ;\|p\|_{E}:=\max \left\{\left\|p_{-}\right\|_{E},\left\|p_{+}\right\|_{E}\right\}$. Если функция $f$ определена на $E$ и принимает, вообще говоря, значения $+\infty$ и $-\infty$, а $p(x)-$ знакочувствительньй вес на $E$, то положим

$$
(f, p)(x):=f^{+}(x) p_{+}(x)-f^{-}(x) p_{-}(x), \quad|f|_{p, E}=\|(f, p)\|_{E}
$$

$($ считаем $0 \cdot( \pm \infty)=( \pm \infty) \cdot 0=0, a \cdot( \pm \infty)=( \pm \infty) \cdot a= \pm \infty$ при $0<a \leqslant+\infty)$. Очевидно, $|f|_{p, E}$ - положительньй сублинейный функционал на линейном пространстве $C(E)$ непрерывных на $E$ функций (вообще говоря, $|-f|_{p, E} \neq|f|_{p, E}$ ). Напомним, что неотрицательньй функционал $P(f)$ на некотором линейном пространстве $\mathscr{L}$ (принимающий и значение $+\infty$ ) называется положительным сублинейным если $P(\alpha f)=\alpha P(f)$ при любых $f \in \mathscr{L}, \alpha>0$ и $P(f+g) \leqslant P(f)+P(g)$ при любых $f, g \in \mathscr{L}$ (см. [7, с. 34], [8, с. 125]).

Уклонением $l \in \mathscr{L}$ от $f \in \mathscr{L}$ относительно неотрицательного функционала $P$ назовем величину $P(l-f)$ (вообще говоря, $P(l-f) \neq P(f-l)$ ); наименьшим уклонением непустого мнохсества $L \subset \mathscr{L}$ от әлемента $f \in \mathscr{L}$ относительно функиионала $P$ назьвается $E(P ; L, f):=\inf \{P(l-f): l \in L\} ; l(P ; L, f)$ обозначает соответствующий әлемент наилучшего приближсения (если он сушествует): $P(l(P ; L, f)-f)=E(P ; L, f)$. При $P(\cdot)=|\cdot|_{p, E}$ пишем $E(p, L, f)$, 
$l(p, L, f)$ вместо $E(P ; L, f), l(P ; L, f)$, соответственно, а приближение относительно функционала $|\cdot|_{p, E}$ называем равномерным приближением со знакочувствительным весом р.

Заметим, что во всех интересующих нас ниже вопросах равномерного приближения со знакочувствительньм весом можно ограничиться лишь полунепрерывными сверху весами. Это следует из того, что для любой непрерывной на компакте $E($ с метрикой $d)$ функции $f$ и любой ограниченной сверху на $E$ функции $p(x)$ имеем

$$
\sup \{f(x) p(x): x \in E\}=\max \{f(x) \tilde{p}(x): x \in E\},
$$

где функция $\tilde{p}(x):=\lim _{\delta \rightarrow 0} \sup \{p(t): d(t, x)<\delta\}$ полунепрерывна сверху (Р. Бэр $[9$, с. 77]).

Отметим еше следующее. Следуя С. Н. Бернштейну [10], систему непрерывных функций $\varphi_{1}(x), \ldots, \varphi_{n}(x), x \in E$, полиномы по которой на множестве $E$ удовлетворяют условию Хаара, часто называют системой Чебышёва или чебышёвской системой на $E$ (см., например, [2, с. 85]), а подпространство $L$, порожденное системой Чебышёва, - чебъиёвским подпространством (см., например, [11, с. 14]). В то же время чебышёвским подпространством принято также называть подпространство банахова пространства, обладаюшее в нем свойством сушествования и единственности (см., например, [12, с. 151]). В контексте рассматриваемой задачи, в которой исследуются свойства сушествования и единственности системы $(p, L)$, удобно придерживаться терминологии, идущей от Бернштейна и связывающей с именем Чебышёва свойство элементов $n$-мерного подпространства $L$ на тех или иных подмножествах иметь не более $n-1$ нулей. С именем Хаара в статье связываются характеристики знакочувствительного веса $p$, обеспечиваюшие указанное свойство на определенных подмножествах (такие, как ядро Хаара веса $p$, хааровский вес и т.п. - см. §3).

\section{§1. Общий критерий единственности}

Пусть функция $f(x)$ определена на некотором множестве $E, M(f)=M(f, E):=$ $\{x \in E: f(x) \neq 0\}$ - носитель функции $f$ на $E, N(f)=N(f, E):=\{x \in E:$ $f(x)=0\}-$ аннулятор функиии $f$ на $E$ (очевидно, $M(f) \cup N(f)=E$ ). Если $L-$ некоторое семейство функций на $E$, то $N(L, E):=\{x \in E: f(x)=0 \forall f \in L\}$. Как обычно, замыкание носителя $M(f)$ функции $f$ обозначаем через $\operatorname{supp} f$ и называем замкнутым носителем функции $f$.

Пусть $p(x)=\left(p_{-}(x), p_{+}(x)\right)$ - знакочувствительный вес на множестве $E$. Будут использованы следующие обозначения и термины: $M(p)=M(p, E)=M\left(p_{-}, E\right) \cup$ $M\left(p_{+}, E\right)$ - носитель веса $p ; F(p)=F(p, E)=M\left(p_{-}, E\right) \cap M\left(p_{+}, E\right)$ - остов веса $p$ (множество точек невырожденности веса $p$ на $E: F(p)=\left\{x \in E: p_{-}(x)>0\right.$, $\left.\left.p_{+}(x)>0\right\}\right) ; M(p) \backslash F(p)$ - множество полувырожденности веса $p$ (очевидно, $M(p) \backslash F(p)$ - симметрическая разность множеств $M\left(p_{-}\right)$и $\left.M\left(p_{+}\right)\right) ; N(p)=$ $N(p, E)=N\left(p_{-}, E\right) \cap N\left(p_{+}, E\right)$ - аннулятор веса $p$ (множество вырожденности веca $p: N(p)=\{x \in E: p(x)=(0,0)\}) ; \operatorname{supp} p:=\operatorname{supp} p_{-} \cup \operatorname{supp} p_{+}=\bar{M}(p, E)-$ замкнутый носитель веса $p ; \Pi(p)=\Pi(p, E):=\operatorname{supp} p_{-} \cap \operatorname{supp} p_{+}-($замкнутое $)$ ядро веса $p ; \Pi^{0}(p)=\Pi^{0}(p, E)$ - открытое ядро веса $p$, т.е. открытое ядро множества $\Pi(p)$. 
Отметим, что замыкание $\bar{F}(p)$ остова $F(p)$ веса $p$ это, вообше говоря, собственное подмножество его ядра П $(p)$ (например, если $R(x)=1 / q$ при $x=s / q$ (дробь несократимая) и $R(x)=0$ при иррациональном $x, p(x)=(R(x), R(x+\sqrt{2}))$, то $F(p, \mathbb{R})=\varnothing, \Pi(p, \mathbb{R})=\mathbb{R} ;$ очевидно, вес $p$ здесь полунепрерывен сверху на $\mathbb{R})$.

Через $\mathscr{P}(E)$ будем обозначать семейство всех конечных нетривиальных $(p(x) \not \equiv(0,0))$ полунепрерывных сверху на множестве $E$ знакочувствительных весов $p=\left(p_{-}, p_{+}\right)$; как обычно, $C(E)$ - пространство непрерывных на некотором множестве $E$ функций с равномерной нормой $\|\cdot\|_{E}$. Всюду ниже в $\S 1$ фраза “ $E-$ компакт" означает, что $E$ - некоторьй произвольный метрический компакт.

Отметим еще, что если $E$ - компакт, $f \in C(E), p \in \mathscr{P}(E),|f|_{p, E}>0$, то множества

$$
\begin{gathered}
X^{-}=\left\{x \in E:-f(x) p_{-}(x)=|f|_{p, E}\right\}, \quad X^{+}=\left\{x \in E: f(x) p_{+}(x)=|f|_{p, E}\right\}, \\
X=\left\{x \in E:|(f, p)(x)|=|f|_{p, E}\right\}=X^{-} \cup X^{+}
\end{gathered}
$$

являются замкнутьми множествами.

Следуюшая теорема является распространением критерия элемента наилучшего приближения А.Н. Колмогорова [13] (доказательство см. также в [11, с. 13]) на равномерные аппроксимации со знакочувствительньп весом. Ее доказательство не требует каких-либо новых идей. Однако ввиду важности теоремы для дальнейшего, оно приводится во всех деталях. Отметим также, что обычно в качестве приближаюшего множества в критерии Колмогорова выступает конечномерное подпространство.

ТЕОРема 1. Пусть $E$ - компакт, $L$ - выпуклое подмножество в $C(E)$, $p \in \mathscr{P}(E), f \in C(E), l_{0} \in L$,

$$
X\left(p, l_{0}\right):=\left\{x \in E:\left|\left(l_{0}-f, p\right)(x)\right|=\left|l_{0}-f\right|_{p, E}>0\right\}
$$

- множсество точек максимального уклонения $l_{0}$ от $f$. Для того чтобь функиия $l_{0}$ бъла әлементом наилучшего приближения для $f$ необходимо и достаточно, чтобь не нашлось такой функиии $l \in L$, что разность $l_{0}(x)-l(x)$ во всех точках $x$ мнохсества $X\left(p, l_{0}\right)$ принимает значения того жсе знака, что и функиия $l_{0}(x)-f(x)$.

ДокАЗАтЕЛЬство. Необходимость. Предположим противное. Пусть $l(x)-$ функция из $L$ такая, что $h(x):=l_{0}(x)-l(x)$ принимает во всех точках множества $X:=X\left(p, l_{0}\right)$ значения тех же знаков, что и $g(x):=l_{0}(x)-f(x)$. Положим $\mu=|h|_{p, E}, \lambda=\min \{|h(x)|: x \in X\}$. Так как множество $X$ замкнуто, то $\lambda>0$. Пусть $\delta>0, X(\delta)$ - пересечение компакта $E$ с открытой $\delta$-окрестностью множества $X$. Возьмем $\delta$ настолько малым, чтобы на $X(\delta)$ функции $g(x)$ и $h(x)$ принимали значения одного знака и чтобы выполнялось неравенство

$$
\inf \{|h(x)|: x \in X(\delta)\} \geqslant \frac{\lambda}{2} .
$$

Поскольку множество $F=E \backslash X(\delta)$ замкнуто и не содержит точек из $X$ (где $\left.X=\left\{x \in E:|(g, p)(x)|=|g|_{p, E}\right\}\right)$, то $|g|_{p, F}<|g|_{p, E}$. 
Положим $\varepsilon=|g|_{p, E}-|g|_{p, F}, m(x)=l_{0}(x)-\alpha h(x), \alpha=\varepsilon /(2 \mu)$. Очевидно, $m=(1-\alpha) l_{0}+\alpha l \in L$. При $x \in F$ имеем

$$
\begin{aligned}
|(m-f, p)(x)| & \leqslant|(g, p)(x)|+\frac{\varepsilon|(h, p)(x)|}{2 \mu} \\
& \leqslant|g|_{p, E}-\varepsilon+\frac{\varepsilon}{2}<|g|_{p, E} .
\end{aligned}
$$

При $x \in X^{+}(\delta):=\left\{x: x \in X(\delta),(g, p)(x)=|g|_{p, E}\right\}$ функции $g(x)$ и $h(x)$ положительны и выполняется неравенство $h(x) \geqslant \lambda / 2$ (см. (2)). Поэтому, если $x \in X^{+}(\delta)$, то

$$
(m(x)-f(x)) p_{+}(x)=\left(g(x)-\frac{\varepsilon h(x)}{2 \mu}\right) p_{+}(x) \leqslant\left(g(x)-\frac{\varepsilon \lambda}{4 \mu}\right) p_{+}(x)<|g|_{p, E} .
$$

Аналогично, при $x \in X^{-}(\delta):=X(\delta) \backslash X^{+}(\delta)$ получим неравенство

$$
-(m(x)-f(x)) p_{-}(x)<|g|_{p, E},
$$

так что $|(m-f, p)(x)|<|g|_{p, E}$ при $x \in X(\delta)$. Итак, во всех точках $x$ компакта $E$, $E=F \cup X(\delta)$, имеем $|(m-f, p)(x)|<\left|l_{0}-f\right|_{p, E}$.

Достаточность. Предположим противное. Пусть $l_{0}$ не является элементом наилучшего приближения для $f$, т.е. $\left|l_{0}-f\right|_{p, E}=E(p, L, f)+\sigma$, где $\sigma>0$. Пусть $l-$ элемент из $L$ такой, что $|l-f|_{p, E} \leqslant E(p, L, f)+\sigma / 2$. Тогда, если $x \in X\left(p, l_{0}\right)$ и $l_{0}(x)-f(x)>0$, то

$$
\left(l_{0}(x)-f(x)\right) p_{+}(x)=\left|l_{0}-f\right|_{p, E}>E(p, L, f)+\frac{\sigma}{2} \geqslant|l-f|_{p, E} \geqslant(l(x)-f(x)) p_{+}(x),
$$

откуда $l_{0}(x)-l(x)>0$. Если $x \in X\left(p, l_{0}\right)$, а $l_{0}(x)-f(x)<0$, то, аналогично, получим $l_{0}(x)-l(x)<0$, так что $l_{0}(x)-l(x)$ во всех точках множества $X\left(p, l_{0}\right)$ принимает значения того же знака, что и $l_{0}(x)-f(x)$. Теорема доказана.

ЗАмЕчАнИЕ 1 . В теореме 1 множество $L$ достаточно считать звездным относительно $l_{0}$, т.е. таким, что если $l \in L, \alpha \in[0,1]$, то $\alpha l+(1-\alpha) l_{0} \in L$.

СлЕДСТВИЕ 1. Если в теореме $1 L$ - n-мерное подпространство, $\left\{\varphi_{1}, \ldots, \varphi_{n}\right\}-$ базис в нем, $\sigma(x):=\operatorname{sign}\left(l_{0}(x)-f(x)\right)$, то для того чтобы функция $l_{0}$ была әлементом наилучшего приближения для $f$ необходимо и достаточно, чтобы была несовместна следующая (вообще говоря, континуальная) система линейных неравенств относительно $c_{i}:$

$$
\sigma(x) \sum_{i=1}^{n} c_{i} \varphi_{i}(x)>0 \quad \text { npu } \quad x \in X\left(p, l_{0}\right) .
$$

Следующая ниже лемма позволяет указанную бесконечную систему неравенств свести к конечной системе. Утверждения подобного рода можно отнести к так называемым теоремам об очистке, нашедшем применение в теории приближений прежде всего в вопросах характеризации элемента наилучшего приближения (см., например, [12, с. 151], [7, с. 120, 164]). 
Лемма А. Пусть $E$ - компакт, $L$ - п-мерное подпространство в $C(E)$ и пусть в $L$ нет функиий, положстельных на E. Тогда найдутся натуральное число $r \leqslant n+1$, точки $x_{1}, \ldots, x_{r} \in E$ и положсительнье числа $\lambda_{1}, \ldots, \lambda_{r}$ такие, что

$$
\sum_{k=1}^{r} \lambda_{k} l\left(x_{k}\right)=0 \quad \forall l \in L .
$$

Таким образом, в L нет функиий, положительных на конечном подмножсестве $X=\left\{x_{1}, \ldots, x_{r}\right\} \subset E, r \leqslant n+1$.

Лемма А отличается от аналогичных известных утверждений, главным образом, формой, приближенной к запросам решаемой задачи (ср. ее, например, с предложением 3.3.5 в [4]). Однако целесообразно привести ее доказательство.

ДокАЗАТЕЛЬСТво ЛЕммы А. Пусть $\Phi(x)=\left(\varphi_{1}(x), \ldots, \varphi_{n}(x)\right)$ - некоторьй базис в $L, \boldsymbol{c}=\left(c_{1}, \ldots, c_{n}\right) \in \mathbb{R}^{n},(\boldsymbol{c}, \Phi(x))=c_{1} \varphi_{1}(x)+\cdots+c_{n} \varphi_{n}(x)$. По условию система неравенств $(\boldsymbol{c}, \Phi(x))>0, x \in E$, несовместна. Это означает, что выпуклая оболочка в $\mathbb{R}^{n}$ (компактного) множества векторов $\Phi(x), x \in E$, содержит нулевой вектор 0. По теореме Каратеодори (см., например, [4, с. 76]) найдутся такие натуральное $r \leqslant n+1$, векторы $\Phi_{k}=\Phi\left(x_{k}\right), k=1, \ldots, r$, из множества $\{\Phi(x): x \in E\}$ и положительные числа $\lambda_{1}, \ldots, \lambda_{r}, \lambda_{1}+\cdots+\lambda_{r}=1$, что $\lambda_{1} \Phi\left(x_{1}\right)+\cdots+\lambda_{r} \Phi\left(x_{r}\right)=\mathbf{0}$. Записанное покоординатно последнее равенство имеет вид $\lambda_{1} \varphi_{i}\left(x_{1}\right)+\cdots+\lambda_{r} \varphi_{i}\left(x_{r}\right)=0, i=1, \ldots, n$, из которого следует, что для любой функции $l(x)=c_{1} \varphi_{1}(x)+\cdots+c_{n} \varphi_{n}(x)$ выполняется (3). Лемма доказана.

Теорема 1 и лемма А позволяют без труда установить критерий элемента наилучшего равномерного приближения со знакочувствительным весом в следующей форме.

Теорема А. Пусть $E$ - компакт, $L-n$-мерное подпространство в $C(E)$, $p \in \mathscr{P}(E), f \in C(E), E(p, L, f)>0$. Для того чтоби әлемент $l_{0} \in L$ бил әлементом наилучшего приближения для функиии $f$ необходимо и достаточно, чтобьи нашлись натуральное число $r \leqslant n+1$, точки $x_{1}, \ldots, x_{r} \in E$, челье числа $\sigma_{k}= \pm 1$ и положстельные числа $\lambda_{k}$, удовлетворяющие условиям:

$$
\begin{aligned}
& \text { а) } \sigma_{k} \cdot\left(l_{0}-f, p\right)\left(x_{k}\right)=\left|l_{0}-f\right|_{p, E}, \quad k=1, \ldots, r ; \\
& \text { б) } \sum_{k=1}^{r} \lambda_{k} \sigma_{k} l\left(x_{k}\right)=0 \quad \forall l \in L .
\end{aligned}
$$

ДокАЗАТЕЛЬство. Необходимость. Пусть $l_{0}$ - элемент наилучшего приближения, множество $X=X\left(p, l_{0}\right)$ определено в теореме 1 равенством $(1), \sigma(x)=$ $\operatorname{sign}\left(l_{0}(x)-f(x)\right), L_{\sigma}-$ семейство всех функций с областью определения $X$, имеющих вид $\sigma(x) l(x)$, где $l \in L$. Очевидно, $L_{\sigma}-$ подпространство пространства $C(X)$ размерности $\leqslant n$. По теореме 1 в $L_{\sigma}$ нет функций, положительных на $X$. Применяя теперь к $L_{\sigma}$ лемму А, получаем "необходимую” часть теоремы А.

Достаточность. Из условий а) и б), очевидно, следует, что в $L$ нет функций, принимающих на множестве $X$ значения того же знака, что и функция $\left(l_{0}-f, p\right)(x)$ или, эквивалентно, функция $l_{0}(x)-f(x)$. Отсюда и из теоремы 1 получаем "достаточную" часть теоремы. Теорема доказана. 
Теорема А может быть получена также из общих теорем выпуклого исчисления, приведенных, например, в $[7$, с. 41,120$]$. Однако такой дедуктивный путь в нашем случае вряд ли будет более прямьм. Теорема А является непосредственным обобшением известного критерия элемента наилучшего равномерного приближения (см., например, [4, с. 92] и [12, с. 157]).

ОПРЕДЕлЕниЕ 1. Пусть $A$ и $L$ - два непустых подмножества линейного пространства $\mathscr{L}, P$ - положительный сублинейный функционал на $\mathscr{L}$. Скажем, что пара $(P ; L)$ является системой (парой) единственности на $A$, если для каждого элемента $f \in A$, у которого элемент наилучшего приближения $l(P ; L, f)$ существует, этот элемент является единственньм, и при этом множество таких $f$ непусто. Пару $(P ; L)$ назовем системой существования и единственности на $A$, если для каждого $f \in A$ элемент наилучшего приближения $l(P ; L, f)$ существует и единственен.

ЛЕмма 1. Пусть $P$ - произвольный полунепрерывный снизу положстельный сублинейный функиионал на линейном нормированном пространстве $\mathscr{L}$, $L$ - замкнутый выпуклый конечномерный конус в $\mathscr{L}, A \subset \mathscr{L}, A \neq \varnothing$. Тогда если $(P ; L)$ - система единственности на $A$, то $(P ; L)$ является системой существования и единственности на $A$. В частности, әто имеет место, если $E$ - компакт, $\mathscr{L}=C(E), L$ - конечномерное подпространство в $C(E)$, $p \in \mathscr{P}(E), P(\cdot)=|\cdot|_{p, E} \cdot$

ДокАЗАтЕльство. Пусть $f^{*}$ некоторый элемент из $A$, у которого существует единственный элемент наилучшего приближения $l\left(P ; L, f^{*}\right)=: l^{*}, l_{0}$ - произвольньй ненулевой элемент из $L$. Тогда

$$
E(P ; L, f) \leqslant P\left(\left(l^{*}+l_{0}\right)-f\right) \leqslant P\left(l^{*}-f\right)+P\left(l_{0}\right)=E(P ; L, f)+P\left(l_{0}\right) .
$$

Очевидно, $P\left(l_{0}\right)>0$ (иначе $l^{*}$ и $l^{*}+l_{0}$ - два различных элемента наилучшего приближения для $f)$.

Пусть теперь $f$ - произвольный элемент из $\mathscr{L}$ (в частности, из $A$ ), $L(f):=$ $\{l-f: l \in L\}$. Тогда $P(g) \rightarrow \infty$ при $g \in L(f),\|g\| \rightarrow \infty(\|\cdot\|-$ норма в $\mathscr{L})$. Действительно, возьмем некоторую последовательность $\left\{g_{k}\right\}_{k=1}^{\infty} \subset L(f),\left\|g_{k}\right\| \rightarrow \infty$, $k \rightarrow \infty$. Очевидно, $g_{k}=l_{k}-f$, где $l_{k} \in L$. Пусть $l_{0}-$ какая-либо предельная точка последовательности $\left\{l_{k} /\left\|l_{k}\right\|\right\}$, так что $l_{0} \in L,\left\|l_{0}\right\|=1$. По сказанному выше $P\left(l_{0}\right)>0$. А так как $l_{0}$ является предельной точкой и для последовательности $\left\{g_{k} /\left\|g_{k}\right\|\right\}$, то $\underline{\lim } P\left(g_{k} /\left\|g_{k}\right\|\right) \geqslant P\left(l_{0}\right)>0$. Отсюда следует, что $P\left(g_{k}\right) \rightarrow \infty$ при $k \rightarrow \infty$, так что $P(g) \rightarrow \infty$ при $\|g\| \rightarrow \infty$ и $g \in L(f)$.

Таким образом, можно воспользоваться следующим простьм замечанием общего характера об условиях сушествования элемента наилучшего приближения:

если $L$ - такое замкнутое множество в линейном нормированном пространстве $\mathscr{L}$ с нормой $\|\cdot\|$, что его пересечение с любым замкнутым шаром в $\mathscr{L}$ является компактом, $f \in \mathscr{L}$, а $P$ - такой полунепрерывный снизу неотрицательный функционал на $\mathscr{L}$, что $P(g) \rightarrow \infty n p u\|g\| \rightarrow \infty, g \in L(f)$, то у $f$ в L имеется, по крайней мере, один элемент наилучшего приближения $l(P ; L, f)$.

Поскольку $(P ; L)$ - система единственности на $A$, то элемент наилучшего приближения $l(P ; L, f)$ является единственньм. Лемма доказана. 
Ниже в качестве множества $A$ будут, наряду с множеством $C(E)$, выступать также множества

$$
\begin{aligned}
C(E, p, L) & :=\{f \in C(E): E(p, L, f)>0\}, \\
C_{0}(E, p, L) & :=\{f \in C(E): E(p, L, f)=0\} \\
(C(E) & \left.=C(E, p, L) \cup C_{0}(E, p, L)\right),
\end{aligned}
$$

что обусловлено спецификой аппроксимаций с вырождающимся на некоторых подмножествах множества $E$ весом $p$.

Вопрос о необходимых и достаточных условиях на пару $(p, L)$, при которых она является системой единственности на $C_{0}(E, p, L)$, решается достаточно просто.

Лемма 2. Пусть $E$ - компакт, $p=\left(p_{-}, p_{+}\right)$- произвольный (не обязательно ограниченный) вес на $E, L$ - конечномерное подпространство в $C(E)$. Тогда для того чтобы пара $(p, L)$ была системой единственности на $C_{0}(E, p, L)$ необходимо и достаточно, чтобъ ядро П $(p, E)$ веса $p$ было непусто, и чтобы $L$ не вирождалосъ на ядре $\Pi(p, E)$ веса $p$, т.е. чтобы не существовало функции $l \in L$ такой, что $\|l\|_{E}=1, l(x)=0 \quad \forall x \in \Pi(p, E)$. Это же условие является также необходимым и достаточным для того, чтобы пара $(p, L)$ была системой существования и единственности на $C_{0}(E, p, L) ;$ в частности, для этого необходимо, чтобы выполнялось неравенство $\operatorname{card} \Pi(p, E) \geqslant \operatorname{dim} L$.

ДоказАтельство. Необходимость. Допустим противное: либо П $(p, E)=\varnothing$, либо $\Pi(p, E) \neq \varnothing$ и сушествует функция $l \in L$ такая, что $0<\|l\|_{E} \leqslant 1, l(x)=0$ $\forall x \in \Pi(p, E)$. Множество всех таких функций $l$ обозначим через $L(\Pi)$ и положим $\omega(\delta):=\sup \{\omega(l, \delta): l \in L(\Pi)\}, \delta \geqslant 0$, где $\omega(l, \delta)$-модуль непрерывности функции $l$ на $E$ (по теореме Арцела $\omega(0+)=0$ ). Определим непрерывную на $\operatorname{supp} p$ функцию $f$ следуюшим образом. Если $\Pi(p, E)=\varnothing$, то полагаем $f(x)=-1$ при $x \in \operatorname{supp} p_{-}$ и $f(x)=1$ при $x \in \operatorname{supp} p_{+}$. Если $\Pi(p, E) \neq \varnothing$, то полагаем $f(x)=-\omega(d(x))$ при $x \in \operatorname{supp} p_{-}$и $f(x)=\omega(d(x))$ при $x \in \operatorname{supp} p_{+}$, где $d(x)$ расстояние от $x$ до П $(p, E)$. Затем возьмем какое-либо непрерывное продолжение $f^{*}$ функции $f \operatorname{csupp} p$ на весь компакт $E$ (см. [14, с. 134]). Возьмем также какой-нибудь элемент $l$ из $L(\Pi)$, если $\Pi(p, E) \neq \varnothing$, и какой-нибудь элемент $l$ из $L, 0<\|l\|_{E} \leqslant 1$, если $\Pi(p, E)=\varnothing$. Тогда в первом случае по определению $\omega(\delta)$ при $-1 \leqslant t \leqslant 1$ и $x \in \operatorname{supp} p$ будет вьполняться неравенство $|t l(x)| \leqslant \omega(d(x))=\left|f^{*}(x)\right|$, а во втором - неравенство $|t l(x)| \leqslant 1=\left|f^{*}(x)\right|$. Поэтому $t l(x)-f^{*}(x) \geqslant 0$ при $x \in \operatorname{supp} p_{-}, t l(x)-f^{*}(x) \leqslant 0$ при $x \in \operatorname{supp} p_{+}$, так что $\left|\left(t l-f^{*}, p\right)(x)\right|=0$ при $x \in E, E\left(p, L, f^{*}\right)=0$, и $t l(x)$ при любом $t \in[-1,1]$ является элементом наилучшего приближения для $f^{*}$ с весом $p$.

Достаточность. Допустим противное: $(p, L)$ не является системой единственности на $C_{0}(E, p, L)$. Это означает, что сушествует функция $f \in C_{0}(E, p, L)$, имеющая два различных элемента наилучшего приближения, которые ввиду их непрерывности и непрерывности $f$ совпадают с $f$, а значит, и друг с другом на $\Pi(p, E)$. Это означает, что $L$ вырождается на $\Pi(p, E)$. Из доказанного и из леммы 1 следует вторая часть леммы 2. Лемма доказана.

Таким образом, задача о необходимых и достаточных условиях, при которых $(p, L)$ является системой сушествования и единственности на $A=C(E)$, сводится к случаю $A=C(E, p, L)$. Именно этому случаю посвяшены дальнейшие результаты 
параграфа. В силу общности задачи их не всегда удается представить в достаточно простой форме и при желании можно рассматривать лишш как вспомогательные. Они будут использованы в $\S 3$.

ОПРЕДЕЛЕНИЕ 2 . Пусть вес $p(x)=\left(p_{-}(x), p_{+}(x)\right)$ определен на множестве $E$, $X \subset M(p, E)$. Сигнатурой веса $p$ на множестве $X$ назовем, вообще говоря, неоднозначную функцию $\sigma(p ; x)$, определенную на множестве $X$, со следующим множеством значений в каждой точке $x \in X: \sigma(p ; x)=\{-1,1\}$, если $p_{-}(x)>0$, $p_{+}(x)>0 ; \sigma(p ; x)=\{-1\}$, если $p_{-}(x)>0, p_{+}(x)=0 ; \sigma(p ; x)=\{1\}$, если $p_{-}(x)=0, p_{+}(x)>0$. Однозначные фиксированные ветви сигнатуры $\sigma(p ; x)$ будем обозначать через $\sigma(x)$. Если $X=\left\{x_{1}, \ldots, x_{m}\right\}$ - конечное упорядоченное множество, то сигнатуру $\sigma(p ; x)$ некоторого веса $p$ на таком множестве $X$ назовем также $\sigma$-вектором, определенным на $X$. Однозначные ветви $\sigma$-вектора будем представлять в виде обычных векторов $\left(\sigma_{1}, \ldots, \sigma_{m}\right)$, где $\sigma_{i}:=\sigma\left(x_{i}\right)$, так что либо $\sigma_{i}=-1$, либо $\sigma_{i}=1$.

Следующая ниже лемма представляет собой обобщение фрагмента доказательства теоремы Хаара (см., например, [2, с. 81]). Отметим еще, что в соответствии с терминологией, принятой в [8, с. 99], множество $A \subset C(E)$ предкомпактно, если его замькание в $C(E)$ - компакт.

Лемма 3. Пусть $E$ - компакт, $X$ - конечное множество на $E, L$ - некоторое множество в $C(E)$ такое, что его подмножество

$$
L_{0}(X)=\left\{l \in L: 0<\|l\|_{E} \leqslant 1, l(x)=0 \quad \forall x \in X\right\}
$$

непусто и предкомпактно, $p \in \mathscr{P}(E), M(p, E) \supset X$, и пусть существует однозначная ветвь $\sigma(x)$ сигнатуры веса $p$ на $X$ такая, что не существует функции $l \in L, y$ которой $\operatorname{sign} l(x)=-\sigma(x)$ при $x \in X$. Тогда найдется функция $f_{0} \in C(E)$ с (непустымм) множеством әлементов наилучшего приближсения, содержащим $L_{0}(X) \cup\{0\}$, и с положстельным наименьшим уклонением $E\left(p, L, f_{0}\right)\left(=\|p\|_{E}\right)$.

ДокАЗАТЕЛЬСтво. Очевидно, достаточно провести доказательство для случая $\|p\|_{E}=1$. Пусть $q_{+}(x)\left(q_{-}(x)\right)$ - любая непрерьвная мажоранта на $E$ функции $p_{+}(x)$ (соответственно, функции $p_{-}(x)$ ), совпадающая с ней в тех точках множества $X$, в которых $\sigma(x)=1$ (соответственно, $\sigma(x)=-1$ ). Такая мажоранта существует, поскольку $X$ - конечное множество. Пусть $x \in E, d(x)$ - расстояние от $x$ до $X, \omega(\delta):=\sup \left\{\omega(l, \delta): l \in L_{0}(X)\right\}(\omega(0+)=0$ - по теореме Арцела), $s(x)=\omega(d(x)), g_{-}(x)=-(1-s(x)) / q_{-}(x), g_{+}(x)=(1-s(x)) / q_{+}(x)$. Возьмем любую функцию $g \in C(E)$ такую, что

1) $g_{-}(x) \leqslant g(x) \leqslant g_{+}(x), x \in E$

2) $g(x)=g_{-}(x)=-1 / p_{-}(x)$ при $\sigma(x)=-1, g(x)=g_{+}(x)=1 / p_{+}(x)$ при $\sigma(x)=1, x \in X$.

Положим $f_{0}(x)=-g(x)$. Если $E\left(p, L, f_{0}\right)<1$, то для некоторой функции $f \in L$ имеем $\left|f-f_{0}\right|_{p, E}<1$,

$$
\frac{-1}{p_{-}(x)}<f(x)+g(x)<\frac{1}{p_{+}(x)} \quad \forall x \in X .
$$


Поэтому, если $\sigma(x)=-1$, то (см. 2)) $-1 / p_{-}(x)=g(x)$ и, следовательно, $f(x)>0$, если же $\sigma(x)=1$, то $1 / p_{+}(x)=g(x)$, и $f(x)<0$. Таким образом, $\operatorname{sign} f(x)=$ $-\sigma(x)$, что противоречит условию леммы. Итак, $E\left(p, L, f_{0}\right) \geqslant 1$.

Возьмем теперь $\forall l \in L_{0}(X) \cup\{0\}$. Тогда при любом $x \in E$ имеем

$$
\left|l(x)-f_{0}(x)\right|_{p} \leqslant|l(x)|_{p}+|g(x)|_{p} \leqslant s(x) \max \left\{p_{-}(x), p_{+}(x)\right\}+1-s(x) \leqslant 1 .
$$

Итак, $l \in L_{0}(X) \cup\{0\}$ является элементом наилучшего приближения для $f_{0}$, $E\left(p, L, f_{0}\right)=1$. Лемма доказана.

Лемма 4. Пусть $E$ - компакт, $L$ - конечномерное подпространство в $C(E), p \in \mathscr{P}(E) . E с л и(p, L)$ является системой единственности на $C(E, p, L)$ или если $C(E, p, L)$ пусто, то для любого замкнутого подмножества $X \subset$ $M(p, E)$ выполняется одно из следующих двух условий:

A) множество $L(X):=\{l \in L \backslash\{0\}: l(x)=0 \quad \forall x \in X\}$ nусто;

В) множество $L(X)$ непусто и для каждой непрерывной на $X$ однозначной ветви $\sigma(x)$ сигнатурь веса $p$ найдется функиия $l \in L$ такая, что $\operatorname{sign} l(x)=-\sigma(x) \quad \forall x \in X$.

ДокАЗАТЕЛЬСТво. Предположим противное. Именно, пусть найдется замкнутое множество $X \subset M(p, E)$ такое, что $L(X)$ непусто и для некоторой непрерывной на $X$ однозначной ветви $\sigma(x)$ сигнатуры веса $p$ в $L$ не существует функций, принимающих на $X$ значения того же знака, что и $-\sigma(x)$. Другими словами, в семействе $\Sigma:=\{-\sigma(x) f(x): f \in L\}$ нет функций всюду положительных на $X$. По лемме А найдется такое конечное подмножество $Y \subset X$, что в $\Sigma$ нет функций положительных на $Y$ или, что то же, в $L$ нет функций, принимаюших на $Y$ значения того же знака, что и $-\sigma(x)$. Поскольку $L_{0}(Y):=\left\{l \in L: 0<\|l\|_{E}<1, l(x)=0 \forall x \in Y\right\}$ - непустое предкомпактное множество, то по лемме 3 получаем, что $C(E, p, L)$ непусто и что $(p, L)$ не является системой единственности на $C(E, p, L)$. Лемма доказана.

Лемма 5. Пусть $E-$ компакт, $L$ - выпуклый конус в $C(E), p \in \mathscr{P}(E) . E c$ ли для любого замкнутого подмножества $X \subset M(p, E)$ выполняется одно из условий А) или В) леммъ 4 , то либо $(p, L)$ является системой единственности на $C(E, p, L)$, либо $C(E, p, L)$ пусто. Eсли $L$-n-мерное подпространство, то множество $X$ достаточно считать состоящим не более чем из $n+1$ точек.

ДокаЗательство. Пусть $C(E, p, L)$ непусто. Если в $C(E, p, L)$ нет функций, у которых элемент наилучшего приближения сушествует, то доказывать нечего. Предположим, что для некоторой функции $g \in C(E, p, L)$ сушествуют в $L$ два различных элемента $l_{1}$ и $l_{2}$ наилучшего приближения. Тогда $\left(l_{1}+l_{2}\right) / 2$ также элемент наилучшего приближения функции $g$. Положив $f=g-\left(l_{1}+l_{2}\right) / 2$, получим функцию $f$, у которой в сдвинутом конусе $L^{*}=L\left(\left(l_{1}+l_{2}\right) / 2\right)$ один из элементов наилучшего приближения $\varphi_{0}=0, \varphi_{0} \in L^{*}$, и есть еше два ненулевых элемента наилучшего приближения $\varphi_{1}=\left(l_{1}-l_{2}\right) / 2$ и $\varphi_{2}=-\varphi_{1}=\left(l_{2}-l_{1}\right) / 2$, $\varphi_{1}, \varphi_{2} \in L^{*}$. Нетрудно видеть, что функции $\varphi_{1}(x)$ и $\varphi_{2}(x)$ равны нулю всюду на множестве $X\left(p, \varphi_{0}\right)$ точек максимального уклонения функции $\varphi_{0}(x) \equiv 0$ от $f(x)$, $X\left(p, \varphi_{0}\right) \subset M(p, E)$. Действительно, пусть $x \in X\left(p, \varphi_{0}\right)$ и пусть, для определенности, $f(x)<0$, так что $-f(x) p_{+}(x)=E\left(p, L^{*}, f\right)=E(p, L, g)>0$. Тогда, если 
$\varphi_{1}(x) \neq 0$, то либо $\varphi_{1}(x)$, либо $\varphi_{2}(x)$ положительно, например, $\varphi_{1}(x)>0$, и мы получим $\left(\varphi_{1}(x)-f(x)\right) p_{+}(x)>E\left(p, L^{*}, f\right)$, что невозможно. Аналогично приходим к противоречию в случае $x \in X\left(p, \varphi_{0}\right), f(x)>0$.

Итак, множество $L^{*}(X)=\left\{l \in L^{*} \backslash\{0\}, l(x)=0 \quad \forall x \in X\left(p, \varphi_{0}\right)\right\}$ непусто. А поскольку $\varphi_{0} \equiv 0$ является элементом наилучшего приближения для $f$, то по теореме 1 не существует в $L^{*}$ функции, принимающей во всех точках $X\left(p, \varphi_{0}\right)$ значения того же знака, что и $f(x)$. Если $L-n$-мерное подпространство, то по лемме А это же свойство выполняется и для подмножества $X \subset X\left(p, \varphi_{0}\right)$, состояшего не более чем из $n+1$ точек. Положив $\sigma(x)=-\operatorname{sign} f(x), x \in X\left(p, \varphi_{0}\right)(x \in X$, если $L-n$-мерное подпространство), получим непрерывную однозначную ветвь сигнатуры веса $p$, для которой не сушествует в $L^{*}$, а значит и в $L\left(L \subset L^{*}\right.$, так как $L-$ вьпуклый конус) функции, принимающей во всех точках множества $X\left(p, \varphi_{0}\right)$ (соответственно, множества $X$, если $L-n$-мерное подпространство) значения того же знака, что и $-\sigma(x)$. Это противоречит условию леммы. Доказательство закончено.

Из леммы 4 и 5 получаем следуюшее утверждение.

Tеорема 2. Пусть $E$ - компакт, $L-n$-мерное подпространство в $C(E)$, $p \in \mathscr{P}(E), C(E, p, L)$ непусто. Для того чтобъ пара $(p, L)$ являлась системой единственности на $C(E, p, L)$ необходимо и достаточно, чтобы для любого подмнохества $X \subset M(p, E)$, состоящего не более чем из $n+1$ точек, выполнялось одно из следующих условий:

A) множество $L(X):=\{l \in L \backslash\{0\}: l(x)=0 \quad \forall x \in X\}$ nусто;

$\left.\mathrm{B}^{*}\right)$ для любой ветви $\sigma(x)$ сигнатуры веса $p$ на $X$ найдется функция $l \in L$ mакая, ито $\operatorname{sign} l(x)=-\sigma(x) \quad \forall x \in X$.

ЗАмечАние 2. Условие А) может выполняться лишш в случае $\operatorname{card} X \geqslant n$.

ЗАмЕчаниЕ 3 . Если для любого множества $X \subset M(p, E)$, $\operatorname{card} X \leqslant n+1$, вьполняется условие $\left.\mathrm{B}^{*}\right)$, то пусто множество функций $f \in C(E, p, L)$, у которых сушествует хотя бы один элемент наилучшего приближения $l_{0}=l(p, L, f)$.

Действительно, пусть $E(p, L, f)>0, X\left(p, l_{0}\right)$ - множество точек максимального уклонения $l_{0}$ от $f, X\left(p, l_{0}\right) \subset M(p, E)$. Тогда $\sigma(x)=\operatorname{sign}\left(l_{0}(x)-f(x)\right)$ $\left(x \in X\left(p, l_{0}\right)\right)$ - однозначная ветвь сигнатуры веса $p$, и по теореме 1 не существует $l \in L$ такой, что $\operatorname{sign} l(x)=\sigma(x) \forall x \in X\left(p, l_{0}\right)$.

ЗАмечАниЕ 4 . Условия на пару $(p, L)$, необходимые для того, чтобы она была системой единственности на $C(E, p, L)$, существенно отличаются от условий, при которых она является системой единственности на $C(E)$.

Приведем пример пары $(p, L)$, которая является системой единственности на $C(E, p, L)$ при пустом ядре П $(p, E)$, так что $(p, L)$ не является системой единственности на $C_{0}(E, p, L)$, а, следовательно, и на $C(E)$ (напомним, что по лемме 2 , если $(p, L)$ - система единственности на $C_{0}(E, p, L)$, то $\left.\operatorname{card} \Pi(p, E) \geqslant \operatorname{dim} L\right)$.

ПримеР 1. Положим $E=[-3,3], p_{-}(x) \equiv 0, p_{+}(-2)=p_{+}(0)=p_{+}(2)=1$, $p_{+}(x)=0$ при $x \neq \pm 2$ и $x \neq 0, \varphi_{1}(x)=x, \varphi_{2}(x)=1-x^{2}, L=\left\{c_{1} \varphi_{1}+c_{2} \varphi_{2}:\right.$ $\left.c_{1}, c_{2} \in \mathbb{R}\right\}$. Очевидно, $M(p, E)=\{-2,0,2\}$. Если $f(x) \equiv-1$, то $l(x) \equiv 0-$ элемент наилучшего приближения для $f$ (так как, очевидно, не существует положительной на $M(p, E)$ функции $l \in L-$ см. теорему 1$)$. Поэтому $E(p, L, f)=1$ и 
$C(E, p, L)$ непусто. Нетрудно также увидеть, что для $X=\{-2,0,2\}, X=\{-2,0\}$, $X=\{0,2\}$ и $X=\{-2,2\}$ выполняется условие A), а для одноточечных множеств $X \subset M(p, E)$ - условие $\left.\mathrm{B}^{*}\right)$ теоремы 2 . По теореме 2 пара $(p, L)$ является системой единственности на $C(E, p, L)$, тогда как $\Pi(p, E)$ пусто.

Приведем еше некоторые простые примеры применения теоремы 2 , которыми в дальнейшем воспользуемся.

ПримеР 2. Пусть $E=[-1,1], \alpha_{i}(x), i=1,2,3,4,-$ произвольные полунепрерывные сверху на $E$ функции, положительные на $(-1,1)$ и равные нулю в точках -1 и 1 лишш в следуюших случаях: $\alpha_{1}(-1)=0, \alpha_{2}(1)=0, \alpha_{3}(-1)=\alpha_{3}(1)=0$ (так что $\alpha_{4}(x)>0 \forall x \in E$ ). Выбирая эти функции в качестве компонент знакочувствительных весов, образуем следующие веса: $p=\left(\alpha_{1}, \alpha_{2}\right), q=\left(\alpha_{3}, \alpha_{4}\right)$, $r=\left(\alpha_{1}, \alpha_{4}\right), s=\left(\alpha_{1}, \alpha_{3}\right)$. Далее, пусть $L_{1}-$ произвольное чебышёвское на $[-1,1)$ подпространство непрерывных функций (см. ниже определение 5), принимаюших на концах отрезка $E$ равные значения (так что подпространство $L_{1}$ уже не является чебышёвским на $E$ ). Напомним, что размерность такого подпространства может быть только нечетной (см., например, [5, с. 52$]$ ). Подпространство $L_{2}$ определим как линейную оболочку функций $\varphi_{1}(x)=x$ и $\varphi_{2}(x)=1-x^{2}, x \in E$. Очевидно, $L_{2}$ является чебьшёвским на промежутках $[-1,1)$ и $(-1,1]$, но не на всем отрезке $E=[-1,1]$. Теорема 2 без труда позволяет проверить, что пары $\left(p, L_{2}\right),\left(q, L_{1}\right)$, $\left(s, L_{1}\right),\left(s, L_{2}\right)$ являются системами единственности на $C(E)$, а пары $\left(p, L_{1}\right),\left(q, L_{2}\right)$, $\left(r, L_{1}\right),\left(r, L_{2}\right)$ не являются.

Число примеров легко увеличить, если воспользоваться следующим замечанием: если $p=\left(p_{-}, p_{+}\right), p^{*}=\left(p_{+}, p_{-}\right)$, то пары $(p, L)$ и $\left(p^{*}, L\right)$ или одновременно являются системами единственности, соответственно, на $C(E, p, L)$ и $C\left(E, p^{*}, L\right)$, или не являются таковыми.

Сделаем теперь некоторые замечания, облегчающие в иных случаях проверку условий А) и В). Прежде всего, дополним определение 2 следующими определениями.

ОПРЕДЕЛЕНИЕ 3 . Если $p(x)$ - невырожденный вес на $X=\left\{x_{1}, \ldots, x_{m}\right\}$ $\left(p_{-}(x)>0, p_{+}(x)>0 \forall x \in X\right)$, то соответствуюший ему $\sigma$-вектор назовем полныцм на $X$. Если $\sigma_{0}-m$-мерный $\sigma$-вектор, одна из координат которого есть одноточечное множество (либо $\{-1\}$, либо $\{1\}$ ), а остальные составляют полный $(m-1)$-мерный $\sigma$-вектор, то $\sigma_{0}$ назовем квазиполным $\sigma$-вектором. Очевидно, квазиполный $\sigma$-вектор соответствует весу $p(x), x \in X$, полувырожденному в единственной точке множества $X$ и невырожденному в остальных его точках.

ОПРеДЕЛЕнИЕ 4. Пусть $A$ - некоторое множество элементов (векторов) $\left(a_{1}, \ldots, a_{m}\right)$ в $\mathbb{R}^{m}$, а $\sigma$ - некоторый $m$-мерный $\sigma$-вектор. Множество $A_{\sigma}$ всех элементов вида $\left(a_{1} \sigma_{1}, \ldots, a_{m} \sigma_{m}\right)$, где $\left(a_{1}, \ldots, a_{m}\right)$ пробегают все множество $A$, а $\left(\sigma_{1}, \ldots, \sigma_{m}\right)$ - все однозначные ветви $\sigma$-вектора $\sigma$, назовем $\sigma$-производныцм от множества $A$.

Очевидно, если $\sigma$ - полный $m$-мерный $\sigma$-вектор, а $A$ - произвольное непустое множество в $\mathbb{R}^{m}$, то в множестве $A_{\sigma}$ найдется элемент, у которого все координаты неотрицательны. Аналогично, если $\sigma$ - квазиполньй $m$-мерньй $\sigma$-вектор, $A-$ множество центрально симметричное в $\mathbb{R}^{m}, A \neq\{0\}$, то в множестве $A_{\sigma}$ найдется ненулевой вектор, у которого все координаты неотрицательны. 
Лемма 6. Пусть $X=\left\{x_{1}, \ldots, x_{m}\right\}, \sigma$ - некоторый $\sigma$-вектор на множестве $X, \varphi_{1}(x), \ldots, \varphi_{n}(x)$ - некоторая система функиий, определенных на $X$, $A$-множсество решений $\left(a_{1}, \ldots, a_{m}\right)$ системь уравнений

$$
\sum_{k=1}^{m} a_{k} \varphi_{i}\left(x_{k}\right)=0, \quad i=1, \ldots, n
$$

$A_{\sigma}-\sigma$-производное множество от $A, r$-ранг системь (4). Для того чтобь система неравенств относительно $c_{i}, i=1, \ldots, n$,

$$
\sigma\left(x_{k}\right) \sum_{i=1}^{n} c_{i} \varphi_{i}\left(x_{k}\right)>0, \quad k=1, \ldots, m,
$$

бъла совместной для любой однозначной ветви $\left(\sigma\left(x_{1}\right), \ldots, \sigma\left(x_{m}\right)\right)=: \sigma(x)$ данного вектора $\sigma$ необходимо и достаточно, чтобы нулевой әлемент был единственным общим әлементом множества $A_{\sigma}$ и неотрицательного ортанта $\left\{\left(a_{1}, \ldots, a_{m}\right): a_{1} \geqslant 0, \ldots, a_{m} \geqslant 0\right\}$. В частности, если $\sigma-$ полный или квазиполный $\sigma$-вектор, то необходимым и достаточным условием совместности системы неравенств (5) является условие $r=m \leqslant n$.

ДокАЗАТЕльство. Согласно альтернативе Фредгольма для линейных алгебраических систем уравнений система неравенств (5) при каждом выборе ветви $\sigma(x)$ многозначного вектора $\sigma$ совместна тогда и только тогда, когда существует $m$-мерный вектор (зависящий от $\sigma(x))$ с положительными координатами, ортогональньй подпространству решений $\left(b_{1}, \ldots, b_{m}\right)$ системы уравнений

$$
\sum_{k=1}^{m} b_{k} \sigma\left(x_{k}\right) \varphi_{i}\left(x_{k}\right)=0, \quad i=1, \ldots, n .
$$

Отсюда и из того, что решения $\left(b_{1}, \ldots, b_{m}\right)$ связаны с решениями $\left(a_{1}, \ldots, a_{m}\right)$ системы (4) равенствами $b_{k}=a_{k} \cdot \sigma\left(x_{k}\right), k=1, \ldots, m$, получим утверждение леммы.

Рассматриваемая задача о необходимых и достаточных условиях на пару $(p, L)$, при которых она является системой единственности, требует небольшого обобшения понятия “чебышёвское пространство". Напомним обшепринятые определения.

ОПРЕДЕЛЕНИЕ 5 . Конечномерноеподпространство $L$ пространства $C(E)$ функций, непрерывных (не обязательно ограниченных) на некотором абстрактном множестве $E$, называется чебышёвским подпространством на $E$, если с $\operatorname{ard} E \geqslant$ $\operatorname{dim} L=: n$ и каждая функция $l \in L$, не тождественная нулю, имеет на $E$ не более $n-1$ нулей. Систему $\left\{\varphi_{k}(x)\right\}_{k=1}^{n}$ линейно независимых функций, $n \geqslant 1$, определенных и непрерьвных на множестве $E, \operatorname{card} E \geqslant n$, назьвают системой Чебышёва на $E$ или чебъиёвской (на $E$ ), если чебышёвским (на $E$ ) является линейное подпространство, порожденное этой системой.

Условимся говорить, что конечномерное подпространство $L$ функций $f$, определенных на множестве $E$, не вырождается на подмножестве $X \subset E$, если его сужение $\left.L\right|_{X}$ на $X$ имеет ту же размерность что и $L:\left.\operatorname{dim} L\right|_{X}=\operatorname{dim} L$. В противном случае будем говорить, что $L$ вырождается на $X$ (в этом и только в этом случае существует не тождественная нулю на $E$ функция $f \in L$, всюду равная нулю 
на $X)$. Очевидно, $n$-мерное подпространство $L \subset C(E)$ является чебышёвским на $E$ тогда и только тогда, когда оно не вырождается на любом подмножестве $X \subset E$, состоящем из $n$ точек. Напомним, что согласно лемме 2 , если $L-n$-мерное подпространство в $C(E)\left(E-\right.$ компакт), $p=\left(p_{-}, p_{+}\right)$- произвольньй (не обязательно ограниченный) вес на $E,(p, L)$ - система единственности на $C(E)$, то сужение подпространства $L$ на $\Pi(p, E)$ также $n$-мерно (так что $\operatorname{card} \Pi(p, E) \geqslant n)$.

ОПРЕДЕЛЕНИЕ 6 . Будем говорить, что $n$-мерное подпространство $L \subset C(E)$ является чебишёвским на подмножестве $X \subset E, \operatorname{card} X \geqslant n$, если $L$ не вырождается на $X$ и его сужение $\left.L\right|_{X}$ является чебьшёвским (на $X$ ).

Наряду с определением 5 в некоторых случаях будем пользоваться определением чебышёвского подпространства, в котором не накладывается ограничение на число точек множества $E, E \neq \varnothing$. В этом определении не предполагается также конечномерность подпространства $L$. Если $\operatorname{dim} L<\infty, \operatorname{card} E \geqslant \operatorname{dim} L$, то это определение совпадает с определением 5.

ОПРЕДЕЛЕНИЕ 7. Назовем подпространство чебышёвским на $E$, если для любого конечного подмножества $X \subset E$ имеем

$$
\operatorname{dim}\left(\left.L\right|_{X}\right)=\min \{\operatorname{dim} L, \operatorname{card} X\}
$$

Лемма 7. Пусть $E$ - компакт, $L$ - конечномерное подпространство в $C(E), \quad p \in \mathscr{P}(E) . \quad$ Если $(p, L)$ является системой единственности на $C(E, p, L)$, то подпространство $L$ является чебъшёвским на каждом мнохестве вида $F(p, E) \cup\{\xi\}$, где $\xi \in M(p, E)$.

ДокаЗАТЕЛЬСтво. Пусть $X=\left\{x_{1}, \ldots, x_{m}\right\} \subset F(p, E) \cup\{\xi\}$. Если выполняется условие А) леммы 4 , то $\operatorname{dim}\left(\left.L\right|_{X}\right)=\operatorname{dim} L$. Если же выполняется условие В), то система неравенств (5) совместна, и поскольку сигнатура $\sigma(p ; x)$ веса $p$ представляет собой либо полный, либо квазиполный $\sigma$-вектор, то по лемме 6 выполняется условие $r=m \leqslant n$, так что $\operatorname{dim}\left(\left.L\right|_{X}\right)=r=m=\operatorname{card} X$. Итак, $\operatorname{dim}\left(\left.L\right|_{X}\right) \leqslant \min \{\operatorname{dim} L, \operatorname{card} X\}$. Лемма доказана.

Лемма 8. Пусть $E$ - компакт, $L$ - конечномерное подпространство в $C(E), p \in \mathscr{P}(E), C(E, p, L) \neq \varnothing$. Если подпространство $L$ является чебишёвским на $M(p, E)$, то $(p, L)$ - система единственности на $C(E, p, L)$.

ДоказАтельство. Пусть $X \subset M(p, E)$. Если $\operatorname{card} X=n+1$, то в обозначениях леммы $4 L(X)=\varnothing$ (по определению чебышёвского подпространства) и вьполняется условие А). Если $\operatorname{card} X \leqslant n$, то на множестве $X$ имеет решение любая интерполяционная задача и выполняется условие В). Остается воспользоваться леммой 5 .

Параграф заключим следующим утверждением.

Теорема 3. Пусть $E$ - компакт, $L$ - n-мерное подпространство в $C(E)$, $p \in \mathscr{P}(E), F(p, E)=M(p, E)=: F, \operatorname{card} F \geqslant n$. Для того чтобъ пара $(p, L)$ бъла системой существования и единственности на $C(E)$ необходимо и достаточно, чтобъ подпространство $L$ бъло чебъиёвским на $F$. 
ДоКАЗАТЕЛЬСТво. Из лемм 7 и 8 получаем, что $L$ - чебышёвское подпространство на $F$ тогда и только тогда, когда $(p, L)$ - система единственности на $C(E, p, L)$. Отсюда, из леммы 2 и леммы 1 следует требуемое. Теорема доказана.

В теореме 3 отражен простейший случай, когда у веса $p$ отсутствуют точки полувырожденности. Общий случай будет рассмотрен в ряде утверждений $§ 3$.

\section{§ 2. Чебышёвское продолжение системы функций}

Пусть система $\Phi=\left\{\varphi_{1}, \ldots, \varphi_{n}\right\}$ непрерьвных на множестве $\Delta \subset \mathbb{R}$ функций $\varphi_{i}(x)$ является системой Чебьшёва на некотором его подмножестве $E$. Скажем, что система $\Phi$ имеет чебъшёвское продолжение на $E_{1} \subset \Delta$, если $E \subset E_{1}$ и $\Phi$ является системой Чебьшёва на $E_{1}$.

В этом параграфе изучаются наиболее общие условия чебьшёвского продолжения, использующие, в основном, свойства участвующих в нем множеств $\left(E, E_{1}\right.$, $\Delta$ и т.п.). Выясняется, что эти условия выражаются, главным образом, через топологические характеристики этих множеств. Впрочем, здесь не всегда бывает возможным избежать использования характеристик и самой системы $\Phi$. В связи с этим наряду с обычным аннулятором системы $\Phi$ вводится, так называемый, хааровский аннулятор системы, совпадающий в некоторых, представляюших интерес для приложений, частных случаях с обычным. Полученные результаты используются в следуюшем параграфе при дальнейшем исследовании проблемы единственности элемента наилучшего равномерного приближения со знакочувствительным весом.

При формулировке и доказательстве лемме 9 и 10 воспользуемся следуюшими обозначениями: если действительнозначная функция $f(x)$ определена на некотором множестве $\Delta, F \subset \Delta, y \in \mathbb{R}$, то

$$
N(f, \Delta ; y):=\{x \in \Delta: f(x)=y\}
$$

$($ так что $N(f, \Delta)=N(f, \Delta ; 0)-$ см. начало $\S 1)$,

$$
Y(f, \Delta, F):=\{y: N(f, \Delta ; y) \neq \varnothing, N(f, \Delta ; y) \subset F\}
$$

в случае, когда функция $f$ фиксирована, используем также обозначения $N(\Delta ; y)$ и $Y(\Delta, F)$ вместо $N(f, \Delta ; y)$ и $Y(f, \Delta, F)$, соответственно.

Лемма 9. Если $f(x)$ - действительнозначная непрерывная на промежутке $\Delta \subset \mathbb{R}$ функция, не постоянная ни на каком интервале $(\alpha, \beta) \subset \Delta, F-$ множество нигде не плотное на $\Delta$, то множество $Y(\Delta, F)$ нигде не плотно на $\mathbb{R}$.

ДокаЗАТЕЛЬСтво. Пусть $y^{*} \in Y:=Y(\Delta, F), x^{*} \in F, f\left(x^{*}\right)=y^{*}$. Тогда в любой окрестности $U=U\left(x^{*}\right)$ точки $x^{*}$ имеется интервал $V=V(U)$, не содержаший точек множества $F$. Поэтому из непрерьвности функции $f$ в точке $x^{*}$ и из ее непостоянства ни на каком интервале следует, что в любой окрестности точки $y^{*}$ имеется невырожденный в точку промежуток $f(V)$ - образ некоторого интервала $V$, не содержашего точек множества $F$. Очевидно, $f(V)$ не содержит точек множества $Y$, так что $Y$ нигде не плотно на $\mathbb{R}$. Лемма доказана. 
Напомним, что множество $E$ на промежутке $\Delta$ называется остаточным на $\Delta$, если $\Delta \backslash E$ является множеством первой категории на $\Delta$, т.е. счетным объединением нигде не плотных на $\Delta$ множеств. Через $f(E)$ будем, как обычно, обозначать множество значений, принимаемых функцией $f$ на множестве $E$.

ЛЕмма 10. Если $f(x)$ - действительнозначная непрерывная на промежутке $\Delta \subset \mathbb{R}$ функиия, не постоянная ни на каком интервале $(\alpha, \beta) \subset \Delta$, a $E-$ остаточное множество на $\Delta$, то $f(E)$ является остаточныц множеством на $f(\Delta)$.

ДоКАЗАТЕЛЬСТво. Пусть множества $F_{n}, n=1,2, \ldots$, нигде не плотны на $\Delta$, $F_{1} \subset F_{2} \subset \cdots, E_{n}=\Delta \backslash F_{n}$,

$$
F=\bigcup_{n=1}^{\infty} F_{n}, \quad E=\bigcap_{n=1}^{\infty} E_{n}=\Delta \backslash F .
$$

Через $J_{n}=\left\{\Delta_{n, k}\right\}$ обозначим множество всех промежутков - назовем их промежутками $n$-го ранга - дополнительных на $\Delta$ к множеству $F_{n}$ (индекс $k$ пробегает конечную или бесконечную последовательность номеров $1,2, \ldots$, зависяшую от $n)$. Пусть $S$ - множество концов всех промежутков $\Delta_{n, k}, n=1,2, \ldots$ Покажем (см. (7), (8)), что

$$
Y(\Delta, F) \backslash f(S) \subset Y\left(\Delta, F_{1}\right) \cup\left(\bigcup_{n, k} Y\left(\Delta_{n, k}, F_{n+1}\right)\right),
$$

где второе объединение берется по всем $n=1,2, \ldots$ и всем $k$ таким, что $\Delta_{n, k} \in J_{n}$. Возьмем $y^{*} \in Y(\Delta, F) \backslash f(S)$. Пусть множество $N\left(\Delta_{1, k} ; y^{*}\right)$ пусто для каждого промежутка $\Delta_{1, k}$. Тогда $N\left(\Delta ; y^{*}\right) \subset F_{1}$, так что $y^{*} \in Y\left(\Delta, F_{1}\right)$. Пусть теперь для некоторого промежутка $\Delta_{1}$ 1-го ранга, $\Delta_{1} \in J_{1}$, множество $N\left(\Delta_{1} ; y^{*}\right)$ непусто. Тогда либо $N\left(\Delta_{1} ; y^{*}\right) \subset F_{2}$ и, тем самым,

$$
y^{*} \in Y\left(\Delta, F_{2}\right) \subset \bigcup_{k} Y\left(\Delta_{1, k}, F_{2}\right),
$$

либо для некоторого промежутка $\Delta_{2}$ 2-го ранга, содержашегося в $\Delta_{1}$, множество $N\left(\Delta_{2}, y^{*}\right)$ непусто. В последнем случае либо $N\left(\Delta_{2} ; y^{*}\right) \subset F_{3}$ и, тем самьм,

$$
y^{*} \in Y\left(\Delta_{2}, F_{3}\right) \subset \bigcup_{k} Y\left(\Delta_{2, k}, F_{3}\right),
$$

либо для некоторого промежутка $\Delta_{3} 3$-го ранга, содержашегося в $\Delta_{2}$, множество $N\left(\Delta_{3} ; y^{*}\right)$ непусто и т.д.

При некотором конечном $m$ получим $\Delta_{1} \subset \Delta_{2} \subset \cdots \subset \Delta_{m}, \Delta_{n} \in J_{n}$,

$$
y^{*} \in Y\left(\Delta_{m}, F_{m+1}\right) \subset \bigcup_{k} Y\left(\Delta_{m, k}, F_{m+1}\right) .
$$

Действительно, пусть такого конечного $m$ нет. Тогда $N\left(\Delta_{n} ; y^{*}\right)$ непусто при каждом $n=1,2, \ldots$ Возьмем какую-нибудь последовательность $\left\{x_{n}\right\}$, $x_{n} \in N\left(\Delta_{n} ; y^{*}\right) \subset \Delta_{n}$. Пусть $x^{*}-$ некоторая ее предельная точка. Тогда 
$f\left(x^{*}\right)=y^{*}$. Так как $y^{*} \notin f(S)$, то точка $x^{*}$ является внутренней для каждого из промежутков $\Delta_{n}$ и потому $x^{*} \subset \bigcap \Delta_{n} \subset E$, что противоречит тому, что $x^{*} \in N\left(\Delta ; y^{*}\right) \subset F=\Delta \backslash E$.

Итак, (10) доказано. По лемме 9 каждое из множеств $Y\left(\Delta, F_{1}\right), Y\left(\Delta_{n, k}, F_{n+1}\right)$ нигде не плотно, а множество $f(S)$, очевидно, не более чем счетно. Поэтому из (10) получаем, что $Y(\Delta, F)=\{y: y \in f(\Delta), N(\Delta ; y) \subset F\}$ - это множество первой категории. Остаточное множество $f(\Delta) \backslash Y(\Delta, F)$, очевидно, совпадает с $f(E)$. Лемма доказана.

Следуюшая лемма является основньм инструментом доказательства теоремы 4 этого параграфа. В ней и всюду ниже слова “достижимая граничная точка открытого множества" означают, что эта точка является концом одного из интервалов, составляющих данное открытое множество (которое предполагается лежащим на $\mathbb{R})$.

ЛЕмма 11. Пусть $\Delta$ - либо некоторое открытое множество $\Delta^{0}$ на $\mathbb{R}$, либо множество, полученное присоединением $\kappa \Delta^{0}$ какой-либо его достижимой граничной точки, $F$ - множество первой категории на $\Delta, f$ и $g$ - функиии, непрерьвные на $\Delta, N(f):=N(f, \Delta), N(g):=N(g, \Delta), k=$ $\operatorname{card}(N(f) \backslash F)<\infty, s=\operatorname{card}((N(f) \backslash N(g)) \cap F)<\infty$. Тогда найдется такое $t \in \mathbb{R}$, иmo $\operatorname{card} N(g+t f, \Delta \backslash F) \geqslant k+s$.

ДокАЗАТЕЛЬСтво. Пусть $p=\operatorname{card}((N(f) \cap N(g)) \backslash F), q=\operatorname{card}(N(f) \backslash N(g))$, $x_{1}, \ldots, x_{q}$ - точки, составляющие множество $N(f) \backslash N(g), x_{1}<x_{2}<\cdots<x_{q}$. Очевидно, что $p+q=k+s$ и $x_{1}, \ldots, x_{q}$ - изолированные точки множества $N(f)$.

Рассмотрим промежутки $\Delta_{2 j-1}=\left[x_{j}-\delta, x_{j}\right), \Delta_{2 j}=\left(x_{j}, x_{j}+\delta\right], \delta>0$, $j=1, \ldots, q$, исключив, возможно, из рассмотрения один из них в случае, если при некотором $s$ окажется, что $x_{s} \in \Delta \backslash \Delta^{0}$. Именно, в этом случае исключим из рассмотрения тот единственньй промежуток $\left[x_{s}-\delta, x_{s}\right)$ или $\left(x_{s}, x_{s}+\delta\right]$, на котором точка $x_{s}$ может оказаться недостижимой со стороны множества $\Delta^{0}$. Выберем $\delta$ настолько мальм, чтобы все оставшиеся промежутки $\Delta_{i}$ лежали на $\Delta$, не пересекались, не содержали точек из $N(f)$, и чтобы на них функция $g(x)$ сохраняла знак. Положим $I=\left\{i: \Delta_{i} \subset \Delta\right\}, G=\bigcup_{i \in I} \Delta_{i}$. Очевидно, $2 q-1 \leqslant \operatorname{card} I \leqslant 2 q$.

На каждом из промежутков $\Delta_{i}, i \in I$, функция $\lambda(x)=g^{2}(x) / f^{2}(x)$ непрерывна, положительна и стремится к $+\infty$, когда $x$, оставаясь на промежутке $\Delta_{i}$, стремится к его открытому концу. Считаем, что $\lambda(x) \not \equiv$ const $=c$ ни на каком интервале $(\alpha, \beta) \subset \Delta$. Действительно, в противном случае $g^{2}(x)-c f^{2}(x)=0$ на бесконечном множестве точек $(\alpha, \beta) \backslash F$, и одна из функций $g(x)-\sqrt{c} f(x), g(x)+\sqrt{c} f(x)$ имеет на $(\alpha, \beta) \backslash F$ бесконечное число нулей. Утверждение леммы в этом случае становится очевидным.

По лемме 10 образ $\lambda\left(\Delta_{i} \backslash F\right)$ каждого из множеств $\Delta_{i} \backslash F, i \in I$, является остаточным множеством на промежутке $\lambda\left(\Delta_{i}\right)=\left[m_{i},+\infty\right), m_{i}:=\min \{\lambda(x)$ : $\left.x \in \Delta_{i}\right\}$. Поэтому множество

$$
\Lambda=\bigcap_{i \in I} \lambda\left(\Delta_{i} \backslash F\right)
$$

является остаточным на $\bigcap \lambda\left(\Delta_{i}\right)=[m,+\infty), m=\max \left\{m_{i}: i \in I\right\}$, а уравнение $g^{2}(x)-\lambda f^{2}(x)=0$ для всякого $\lambda \in \Lambda$ имеет на каждом из множеств $\Delta_{i} \backslash F, i \in I$, по крайней мере, один корень. 
Таким образом, общее число корней уравнения $g^{2}(x)-\lambda f^{2}(x)=0$ на множестве $G \backslash F$ или, что то же, уравнения $(g(x)-\sqrt{\lambda} f(x)) \cdot(g(x)+\sqrt{\lambda} f(x))=0$ на том же множестве не менее сard $I$. При этом на множестве $(N(f) \cap N(g)) \backslash F$ (не пересекающемся, очевидно, с $G \backslash F)$ каждый из сомножителей $g(x)-\sqrt{\lambda} f(x), g(x)+\sqrt{\lambda} f(x)$ имеет еще $p$ нулей. Поскольку саrd $I \geqslant 2 q-1$, то одна из функций $g(x)-\sqrt{\lambda} f(x)$, $g(x)+\sqrt{\lambda} f(x)$ имеет не менее $q$ нулей на $G \backslash F$ и не менее $p+q=k+s$ нулей на $\Delta \backslash F$. Лемма доказана.

ОПРЕДЕЛЕНИЕ 8. Пусть $E \subset \Delta \subset \mathbb{R}, \Phi=\left\{\varphi_{1}, \ldots, \varphi_{n}\right\}$ - система непрерывных на $\Delta$ функций, чебышёвская на $E$. Через $N(\Phi, \Delta, E)$ обозначим множество точек $x_{0} \in \Delta \backslash E$ таких, что для каждой точки $x_{0}$ найдется полином по системе $\Phi$, имеющий нуль в точке $x_{0}$ и $n-1$ нулей на $E$. Множество $N(\Phi, \Delta, E)$ назовем хааровским аннулятором системь $\Phi$ на $\Delta \backslash F$.

Отметим следуюшие очевидные свойства хааровского аннулятора:

1) $\Delta \backslash N(\Phi, \Delta, E)$ - это максимально широкое подмножество множества $\Delta$, на котором система $\Phi$ может быть чебышёвской;

2) $N(\Phi, \Delta, E)$ содержит в себе (обычный) аннулятор

$$
N(\Phi, \Delta):=\left\{x \in \Delta: \varphi_{1}(x)=\varphi_{2}(x)=\cdots=\varphi_{n}(x)=0\right\}
$$

3) если $E \subset \Delta_{1} \subset \Delta$, то $N\left(\Phi, \Delta_{1}, E\right) \subset N(\Phi, \Delta, E)$;

4) если $E_{1} \subset E \subset \Delta$, то $N\left(\Phi, \Delta, E_{1}\right) \subset N(\Phi, \Delta, E)$.

В качестве простейшей иллюстрации к этому определению рассмотрим систему $\Phi=\left\{x, 1-x^{2}\right\}, x \in \Delta=[-1,1]$. Тогда, если $E=(-1,1]$, то $N(\Phi, \Delta, E)=\{-1\}$, если $E=[-1,1)$, то $N(\Phi, \Delta, E)=\{1\}$, если же $E=(-a, a)$, где $0<a \leqslant 1$, то $N(\Phi, \Delta, E)=\varnothing$.

Заметим, что далеко не всегда существует чебьшёвское продолжение системы $\Phi$ с $E$ на $E^{*}$, для которого $E^{*} \cup N\left(\Phi, \Delta, E^{*}\right)=\Delta$. Ниже будут указаны некоторые условия, когда такое продолжение существует (теорема 4 ).

Следующая лемма показывает, что в одном нетривиальном частном случае, важном для дальнейших приложений, хааровский аннулятор устроен очень просто.

ЛЕмма 12. Если $\Delta$ - либо некоторое открытое множество $\Delta^{0}$ на $\mathbb{R}$, либо множество, полученное присоединением $\kappa \Delta^{0}$ какой-либо его достижимой граничной точки, $E \subset \Delta, F:=\Delta \backslash E$ - множество первой категории, $\Phi=\left\{\varphi_{1}, \ldots, \varphi_{n}\right\}-$ система непрерьвных на $\Delta$ функиий, чебышёвская на $E$, mo $N(\Phi, \Delta, E)=N(\Phi, \Delta)$.

ДоказАтельство. Достаточно показать, что $N:=N(\Phi, \Delta, E) \subset N(\Phi, \Delta)$. Пусть $N$ не пусто, $x_{0} \in N$, и $f$ такой полином, что $f\left(x_{0}\right)=f\left(x_{1}\right)=\cdots=$ $f\left(x_{n-1}\right)=0$, где $\left\{x_{1}, \ldots, x_{n-1}\right\} \subset E, x_{i} \neq x_{j}$ при $i \neq j$. Такой полином существует по определению множества $N(\Phi, \Delta, E)$. Пусть $g$ - произвольный полином. Предположим, что $g\left(x_{0}\right) \neq 0$. Поскольку $\operatorname{card}(N(f) \backslash F)=n-1$, $\operatorname{card}((N(f) \backslash N(g)) \cap F) \geqslant \operatorname{card}\left(\left\{x_{0}\right\}\right)=1$, то по лемме 11 полином $g+t f$ при некотором $t \in \mathbb{R}$ имеет на $\Delta \backslash F=E$ не менее $(n-1)+1$ нулей, что невозможно. Лемма доказана. 
ЗАмечание 5 . Обратим внимание на то, что в лемме 12 условие $E \subset \Delta$ весьма существенно, и что при непустом $E \backslash \Delta$ равенство $N(\Phi, \Delta, E)=N(\Phi, \Delta)$ не может быть обобщено до равенства $N(\Phi, \Delta \cup E, E)=N(\Phi, \Delta \cup E)$. Действительно, пусть $\Delta=(-1,1), E=(-1,0) \cup(0,1) \cup\{2\}, \Phi_{1}=\{1, x(2-x)\}$. Тогда $\Phi_{1}$ - система непрерывытх на $\Delta \cup E$ функций, чебышёвская на $E, N(\Phi, \Delta \cup E, E)=\{0\}$, $N\left(\Phi_{1}, \Delta \cup E\right)=\varnothing$. Таким образом, даже в случае, когда $\Delta \backslash E$ и $E \backslash \Delta$ это одноточечные множества, указанное обобщение, вообще говоря, невозможно.

Еще один пример: $\Delta=(-1,1 / 2), E=(-1,0) \cup(0,1 / 2) \cup\{1\} \cup\{2\}$, $\Phi_{2}=\{1, x, x(1-x)(2-x)\}$. Здесь $\Phi_{2}$ - система непрерывных на $\Delta \cup E$ функций, чебышёвская на $E, N\left(\Phi_{2}, \Delta \cup E, E\right)=\{0\}, N\left(\Phi_{2}, \Delta \cup E\right)=\varnothing$.

Эти примеры поясняют также роль, которую хааровский аннулятор играет в следуюшей ниже теореме 4. Для ее формулировки воспользуемся понятиями, введенными Бэром в [15].

Говорят, что множество $A$ (любого топологического пространства) обладает свойством Бәра, если сушествует открытое множество $G$ такое, что $A \backslash G$ и $G \backslash A$ - множества первой категории. Если множество $A$ обладает свойством Бэра, то в качестве $G$ может быть взято регулярное открытое множество, т.е. множество, совпадаюшее с открытьм ядром своего замыкания. Это множество единственно и является максимальным из открытых множеств $G$, участвующих в определении множества $A$ со свойством Бэра (см. $[16$, с. 40,41$])$. Обозначим его через $B$ и назовем ядром Бәра множества $A$.

Роль свойства Бэра в топологии аналогична роли измеримости (множеств или функций) в анализе. Ниже эта роль естественньм образом проявляется также в задаче чебышёвского продолжения системы функций.

Tеорема 4. Пусть множество $E$ на $\mathbb{R}$ обладает свойством Бәра, В либо ядро Бәра множества $E$, либо ядро Бәра с присоединенной к нему какой-либо его достижимой граничной точкой, $\Delta:=E \cup B, \Phi=\left\{\varphi_{1}, \ldots, \varphi_{n}\right\}$ - система непрерывных на $\Delta$ функций, чебышёвская на $E, N:=N(\Phi, \Delta, E)$ хааровский аннулятор системв $\Phi$ на $\Delta \backslash E$. Тогда $\Phi$ - система Чебышёва на $E \cup(B \backslash N)$. В частности, если $E \subset B$, то $\Phi$ - система Чебъшёва на $B \backslash N(\Phi, B)$.

ДоКАЗАТЕЛЬСтво. Предположим противное: пусть сушествует полином $f \mathrm{c} n$ нулями $x_{1}, \ldots, x_{n}$ на $E \cup(B \backslash N)=(B \backslash N) \cup P$, где $P:=E \backslash B$. Пусть при этом $n-\nu$, $1 \leqslant \nu \leqslant n$, из них, для определенности, $x_{\nu+1}, \ldots, x_{n}$ лежат на $P(\nu \neq 0$, поскольку на $P$ система $\Phi$ - чебышёвская). Возьмем произвольный полином $g$ с $n-1$ нулями на $E$, в том числе с нулями в точках $x_{\nu+1}, \ldots, x_{n}$, такой полином существует в силу чебышёвости $\Phi$ на $E$. Очевидно, полином $g$ не равен нулю в тех точках из множества $\left\{x_{1}, \ldots, x_{\nu}\right\}$, которые лежат на $F:=B \backslash E$ - иначе эти точки были бы точками хааровского аннулятора $N$. Обозначим через $k$ число нулей полинома $f$ на $E \cap B=B \backslash F$, через $s$ - число точек на $N(f) \cap F$, в которых полином $g$ не равен нулю, - их по сказанному не менее $\nu-k, N(f)=N(f, \Delta)$ - множество нулей функции $f$ на $\Delta$. Вспомним теперь, что $F$ - множество первой категории. По лемме 11 найдется полином вида $g+t f$, имеющий на $B \backslash F=E \cap B$ не менее $k+s \geqslant$ $k+(\nu-k)=\nu$ нулей. Общее число нулей этого полинома на $E=(E \cap B) \cup P$ не менее $\nu+(n-\nu)=n$, что невозможно. Итак, $\Phi$ - система Чебышёва на $(B \backslash N) \cup P$. 
Если $E \subset B$, т.е. $\Delta=B$, то по лемме $12 N=N(\Phi, B, E)=N(\Phi, B)$, так что $E \cup(B \backslash N)=B \backslash N(\Phi, B)$. Теорема доказана.

Установим еше одну теорему о чебышёвском продолжении, но уже несколько иной природы, чем теорема 4.

Tеорема 5. Пусть $G$ и $F$ два множества на $\mathbb{R}, G$ - открытое, $F$ - плотное на $G ; \Phi=\left\{\varphi_{1}, \ldots, \varphi_{n}\right\}$ - система непрерывных на $G \cup F$ функиий, чебышёвская на $F$. При этих условиях $\Phi$ является системой Чебышёва на $G \cup F$ тогда и только тогда, когда пуст ее хааровский аннулятор $N(\Phi, G \cup F, F)$, т.е. когда любой полином по системе $\Phi$, имеющий $n-1$ нулей на $F$, не имеет нулей на $G \backslash F$.

Доказательству предпошлем лемму.

Лемма 13. Пусть $G$ и $F$ два множества на $\mathbb{R}, G$ - открытое, $F$ - плотное на $G, \Phi=\left\{\varphi_{1}, \ldots, \varphi_{n}\right\}$ - система непрерьвных на $G \cup F$ функций, чебышёвская на $F$ и такая, что любой полином $\varphi$ по системе $\Phi$, имеющий $n-1$ нулей на $F$, не имеет нулей на $G \backslash F$. Тогда все нули каждого такого полинома $\varphi$, лехащие на $F \cap G$, нечетные.

ДокАЗАтЕльство. Следует лишш проследить за тем, что при доказательстве хорошо известного частного случая $F=G=(a, b)$ этой леммы (см., например, $[17$, c. 22,23$])$ фактически используется только непрерьвность полиномов на $G$ и плотность множества $F$ на $G$.

ДоКАЗАТЕЛЬСТво ТЕОРЕМЫ 5. 1) Покажем вначале, что, каковы бы ни были точки $x_{1}, \ldots, x_{\nu} \in G$ и $x_{\nu+1}, \ldots, x_{n-1} \in F \backslash G, 0 \leqslant \nu \leqslant n-1$, найдется полином $\varphi$ с нулями в этих точках такой, что все его нечетные нули, лежащие на $G$, исчерпываются точками $x_{1}, \ldots, x_{\nu}$ (априори пока допускаются у $\varphi$ четные нули в других точках множества $G$ ). Для этого возьмем последовательность векторов $\left(x_{1}(k), \ldots, x_{\nu}(k)\right) \rightarrow\left(x_{1}, \ldots, x_{\nu}\right), k \rightarrow \infty$, с координатами $x_{i}(k) \in F$. Положим

$$
\begin{aligned}
& \varphi(k, x)=\lambda(k) \cdot\left|\begin{array}{ccccccc}
\varphi_{1}(x) & \varphi_{1}\left(x_{1}(k)\right) & \ldots & \varphi_{1}\left(x_{\nu}(k)\right) & \varphi_{1}\left(x_{\nu+1}\right) & \ldots & \varphi_{1}\left(x_{n-1}\right) \\
\vdots & \vdots & & \vdots & \vdots & & \vdots \\
\varphi_{n}(x) & \varphi_{n}\left(x_{1}(k)\right) & \ldots & \varphi_{n}\left(x_{\nu}(k)\right) & \varphi_{n}\left(x_{\nu+1}\right) & \ldots & \varphi_{n}\left(x_{n-1}\right)
\end{array}\right| \\
& =\sum_{i=1}^{n} a_{i}(k) \varphi_{i}(x),
\end{aligned}
$$

где $\lambda(k)$ - нормирующий множитель такой, что $a_{1}(k)^{2}+\cdots+a_{n}(k)^{2}=1$. Очевидно, $\varphi(k, x)$ - полином с нулями в точках $x_{1}(k), \ldots, x_{\nu}(k), x_{\nu+1}, \ldots, x_{n-1}$. По условию теоремы $\varphi(k, x)$ других нулей на $G \cup F$ не имеет. По лемме 13 его нули, лежащие на $F \cap G$, нечетные. Пусть вектор $\left(a_{1}, \ldots, a_{n}\right)$ - предельный для последовательности $\left(a_{1}(k), \ldots, a_{n}(k)\right), k=1,2, \ldots$. Тогда полином $\varphi(x)=a_{1} \varphi_{1}(x)+\cdots+a_{n} \varphi_{n}(x)-$ искомый. Действительно, при каждом $j=1, \ldots, \nu$ имеем

$$
\begin{aligned}
\varphi\left(x_{j}\right) & =\sum_{i=1}^{n} a_{i} \varphi_{i}\left(x_{j}\right)=\sum_{i=1}^{n}\left(a_{i}-a_{i}(k)\right) \varphi_{i}\left(x_{j}\right)+\sum_{i=1}^{n} a_{i}(k) \varphi_{i}\left(x_{j}\right) \\
& =\sum_{i=1}^{n}\left(a_{i}-a_{i}(k)\right) \varphi_{i}\left(x_{j}\right) \rightarrow 0
\end{aligned}
$$


при $k \rightarrow \infty$, так что $\varphi\left(x_{j}\right)=0$ при всех $j=1, \ldots, n-1$. Из нечетности нулей $x_{j}(k)$, $j=1, \ldots, \nu$, приближающих полиномов $\varphi(k, x)$ и того, что их у $\varphi(k, x)$ ровно $\nu$ на $G$, следует, очевидно, нечетность нулей $x_{j}, j=1, \ldots, \nu$, и отсутствие у $\varphi(x)$ на $G$ других нечетных нулей.

2) Предположим, что существует нетривиальный полином $f(x)=b_{1} \varphi_{1}(x)+$ $\cdots+b_{n} \varphi_{n}(x)$ с нулями $z_{1}, \ldots, z_{m}, m \geqslant n$, на $G \cup F$, и что ранг системы векторов $\left\{\Phi\left(z_{1}\right), \ldots, \Phi\left(z_{m}\right)\right\}$ равен $n-1$. Пусть $z_{1}, \ldots, z_{s} \in G, z_{s+1}, \ldots, z_{s+p} \in$ $F \backslash G, s+p=m$. Очевидно, $0 \leqslant p \leqslant n-2$, так что $s \geqslant 2$. Выберем в системе $\left\{\Phi\left(z_{1}\right), \ldots, \Phi\left(z_{m}\right)\right\}$ максимальную линейно независимую подсистему векторов так, чтобы в нее вошли векторы $\Phi\left(z_{s+1}\right), \ldots, \Phi\left(z_{s+p}\right)$ (они, очевидно, образуют линейно независимую систему) и еше $\nu=n-1-p, 1 \leqslant \nu \leqslant n-1, \nu<s$, векторов из множества $\left\{\Phi\left(z_{1}\right), \ldots, \Phi\left(z_{s}\right)\right\}$. Пусть, для определенности, это векторы $\Phi\left(z_{1}\right), \ldots, \Phi\left(z_{\nu}\right)$. Аналогично тому, как по точкам $x_{1}, \ldots, x_{\nu}, x_{\nu+1}, \ldots, x_{n-1}$ в п. 1$)$ доказательства был найден полином $\varphi$, найдем по точкам $z_{1}, \ldots, z_{\nu}, z_{s+1}, \ldots, z_{s+p}$, $\nu+p=n-1$, полином $\varphi_{1}$. Поскольку $\left\{\Phi\left(z_{1}\right), \ldots, \Phi\left(z_{\nu}\right), \Phi\left(z_{s+1}\right), \ldots, \Phi\left(z_{s+p}\right)\right\}-$ линейно независимая система из $n-1$ векторов, то полином $\varphi_{1}$ определяется с точностью до множителя, и поэтому с точностью до множителя совпадает с полиномом $f$, коэффициенты которого образуют вектор $\left(b_{1}, \ldots, b_{n}\right.$ ) (также находящийся с точностью до множителя), ортогональный векторам $\Phi\left(z_{1}\right), \ldots, \Phi\left(z_{m}\right)$. Полином $\varphi_{1}$ имеет нечетные нули в точках $z_{1}, \ldots, z_{\nu}$ и четные в точках $z_{\nu+1}, \ldots, z_{s}$, лежащих на $G$ (напомним, что $\nu+1 \leqslant s$ ). Поэтому $z_{1}, \ldots, z_{\nu}$ - нечетные нули полинома $f$, а $z_{\nu+1}, \ldots, z_{s}$ - четные.

Выберем теперь в системе векторов $\left\{\Phi\left(z_{1}\right), \ldots, \Phi\left(z_{m}\right)\right\}$ максимальную линейно независимую подсистему так, чтобы в нее вошел вектор $\Phi\left(z_{\nu+1}\right)$, а в остальном произвольно. Аргументы векторов выбранной подсистемы составляют некоторый набор $n-1$ точек, взятых из множества $\left\{z_{1}, \ldots, z_{m}\right\}$, в который войдет и точка $z_{\nu+1} \in G$. По этому набору найдем полином $\varphi_{2}$ аналогично тому, как по набору точек $z_{1}, \ldots, z_{\nu}, z_{s+1}, \ldots, z_{s+p}$ был найден полином $\varphi_{1}$. Для $\varphi_{2}$ точка $z_{\nu+1}-$ нечетный нуль. Поэтому $z_{\nu+1}-$ нечетный нуль и для полинома $f$. Противоречие говорит о том, что предположение п. 2) не имеет места.

3) Предположим теперь, что для полинома $f(x)=b_{1} \varphi_{1}(x)+\cdots+b_{n} \varphi_{n}(x)$ с нулями $z_{1}, \ldots, z_{m}, m \geqslant n$, на $G \cup F$ ранг $r$ системы $\left\{\Phi\left(z_{1}\right), \ldots, \Phi\left(z_{m}\right)\right\}$ не превосходит $n-2$. Возьмем на $F$ точки $y_{1}, \ldots, y_{n-1-r}$ так, чтобы ранг системы векторов $\left\{\Phi\left(z_{1}\right), \ldots, \Phi\left(z_{m}\right), \Phi\left(y_{1}\right), \ldots, \Phi\left(y_{n-1-r}\right)\right\}$ равнялся $n-1$ (это, очевидно, можно сделать). Пусть $g$ - полином, коэффициенты которого образуют вектор, ортогональный всем векторам вновь образованной системы. Очевидно, точки $z_{1}, \ldots, z_{m}$, $y_{1}, \ldots, y_{n-1-r}-$ это нули полинома $g$. Тем самым, для функции $g$ выполняются те же предположения, которые были сделаны для функции $f$ в п. 2), и противоречивость которых вьше была установлена. Теорема доказана.

В связи с теоремой 5 возникает вопрос: не является ли хааровский аннулятор $N$ системы $\Phi$, чебышёвской на множестве $F$, той естественной границей, вплоть до которой система $\Phi$ всегда имеет чебьшёвское продолжение при условии, что множество $F$ всюду плотно в некоторой окрестности $U$ хааровского аннулятора? Точнее, обязана ли $\Phi$ быть системой Чебышёва на множестве $U \backslash N$ ? Теорема 5 дает положительный ответ на этот вопрос в случае $N=\Phi$. Из доказываемой далее леммы 16 следует отрицательный ответ на этот вопрос в общем случае $(N \neq \varnothing)-$ см. ниже следствие 2. 
Лемме 16 предпошлем две леммы.

Лемма 14. Пусть $А$ и В нигде не плотные множсества на интервале $(0,1), I=[0,1], H$ - множество автоморфизмов отрезка I, оставляющих неподвижними его концы. Тогда для любого $g \in H$ и любого $\varepsilon>0$ найдется автоморфизм $h \in H$ такой, что множества $A$ и $h(B)$ не пересекаются $u$ $\|g-h\|_{I}<\varepsilon$.

ДокАЗАТЕЛЬСТво. Не ограничивая общности, можно считать множества $A$ и $B$ замкнутьми. Определим $\delta$ из равенства $\omega(g, \delta)=\varepsilon$ и разобьем отрезок $I$ на конечное число промежутков $I_{i}$ длины $\left|I_{i}\right| \leqslant \delta$ так, чтобы их концы не лежали на $B$. Положим $J_{i}=g\left(I_{i}\right)$; очевидно, $\left|J_{i}\right| \leqslant \varepsilon$. Через $G_{i}$ обозначим наименьший отрезок, содержащий $g\left(B \cap I_{i}\right)$. Очевидно, $G_{i}$ лежит строго внутри $J_{i}$. Также строго внутри $J_{i}$ возьмем какой-нибудь отрезок $F_{i}$, не содержаший точек множества $A$. Пусть $\varphi_{i}$ - кусочно-линейный гомеоморфизм отрезка $J_{i}$ на себя, оставляющий неподвижньпи концы и отображающий $G_{i}$ на $F_{i}$. Все вместе эти $\varphi_{i}$ определяют некоторое отображение $\varphi \in H$. Автоморфизм $h=\varphi \circ g$ обладает нужными свойствами: $h(B)=\varphi \circ g(B) \subset(0,1) \backslash A,\|g-h\|_{I}<\varepsilon$. Лемма доказана.

Лемма 15. Пусть $A$ и В - множества первой категории на интервале $(0,1)$. Тогда существует автоморфизм $h$ отрезка $I=[0,1]$, оставляющий неподвижными его концы, такой, что $A$ и $h(B)$ не пересекаются.

ДокАЗАТЕЛЬство. Возьмемна $(0,1)$ возрастающие последовательности замкнутых нигдене плотных множеств $\left\{A_{n}\right\}$ и $\left\{B_{n}\right\}$ такие, что $A \subset \bigcup_{n} A_{n}, B \subset \bigcup_{n} B_{n}$. Пусть $H$ - множество автоморфизмов отрезка $I$, оставляющих неподвижными его концы. Известно, что $H$ является $G_{\delta}$-множеством в $C(I)$ и топологически полно, т.е. становится полным после некоторой метризации (см. [16, с. 85, 89], [14, с. 419]).

Положим $H_{n}:=\left\{h \in H: A_{n} \cap h\left(B_{n}\right)=\varnothing\right\}$. Нетрудно увидеть, что $H_{n}-$ множество, открытое в пространстве $C(I)$. По лемме $14 H_{n}$ плотно в $H$. Следовательно, множество $H^{*}=\bigcap_{n} H_{n}$ является остаточным в $H$. Возьмем $h \in H^{*}$. Тогда для любого $n$ имеем $h \in H_{n}$, так что $A_{n} \cap h\left(B_{n}\right)=\varnothing$. Отсюда без труда заключаем, что $A \cap h(B)=\varnothing$. Лемма доказана.

Лемма 16. Пусть $-\infty<a<\alpha<\beta<b<\infty, F-$ произвольное множество первой категории, лехсащее на $(\alpha, \beta), k$ - натуральное число. Тогда существует непрерывная на $\Delta=[a, b]$ функиия $\varphi(x)$ такая, что уравнение $\varphi(x)=$ с при любом $c \in \mathbb{R}$ имеет не более одного корня на $F \cup(\Delta \backslash(\alpha, \beta))$ и не менее $k$ корней на $[\alpha, \beta]$ при $c \in(0,1)$.

ДокАЗАТЕльство. Пусть $k \geqslant 3, n-$ любое четное число такое, что $n \geqslant k-1$, $x_{1}, \ldots, x_{n}$ - точки, не лежащие на $F$, такие, что $\alpha=$ : $x_{0}<x_{1}<\cdots<x_{n}<$ $x_{n+1}:=\beta$. Определим непрерывную на отрезке $\Delta=[a, b]$ функцию $\psi(x)$ так, чтобы она была линейной на каждом из отрезков $\left[a, x_{1}\right],\left[x_{1}, x_{2}\right], \ldots,\left[x_{n-1}, x_{n}\right]$, $\left[x_{n}, b\right]$ и принимала следующие значения: $\psi(\alpha)=0, \psi\left(x_{1}\right)=1, \psi\left(x_{2}\right)=0, \ldots$, $\psi\left(x_{n-1}\right)=1, \psi\left(x_{n}\right)=0, \psi(\beta)=1$. Положим $E_{i}=\psi\left(F \cap\left[x_{i-1}, x_{i}\right]\right), i=$ $1, \ldots, n+1, h_{1}(x) \equiv x$. Очевидно, $h_{1}\left(E_{1}\right)=E_{1}$.

По лемме 15 существует такой автоморфизм $h_{2}$ отрезка $[0,1]$, оставляющий неподвижными его концы, что $h_{1}\left(E_{1}\right) \cap h_{2}\left(E_{2}\right)=\varnothing$. Аналогично, сушествует автоморфизм $h_{3}$ отрезка $[0,1]$ такой, что $\left(h_{1}\left(E_{1}\right) \cup h_{2}\left(E_{2}\right)\right) \cap h_{3}\left(E_{3}\right)=\varnothing$ и т. д. Автоморфизм $h_{n+1}$ отрезка $[0,1]$, оставляющий неподвижными точки 0 и 1 , определим 
условием

$$
\left(h_{1}\left(E_{1}\right) \cup \cdots \cup h_{n}\left(E_{n}\right)\right) \cap h_{n+1}\left(E_{n+1}\right)=\varnothing .
$$

Положим $\varphi(x)=\psi(x)$ при $x \in[a, \alpha], \varphi(x)=h_{i} \circ \psi(x)$ при $x \in\left[x_{i-1}, x_{i}\right]$, $i=1, \ldots, n+1, \varphi(x)=\psi(x)$ при $x \in[\beta, b]$. Очевидно, $\varphi(F)=h_{1}\left(E_{1}\right) \cup h_{2}\left(E_{2}\right) \cup$ $\cdots \cup h_{n+1}\left(E_{n+1}\right) \subset(0,1), h_{i}\left(E_{i}\right) \cap h_{j}\left(E_{j}\right)=\varnothing, i \neq j$. Пусть $c \in(0,1)$. Если при этом $c \in \varphi(F)$, то $c$ принадлежит только одному из множеств $h_{i}\left(E_{i}\right)$, и поэтому уравнение $\varphi(x)=c$ имеет единственньй корень на $F$. Если же $c \in(0,1) \backslash \varphi(F)$, то уравнение $\varphi(x)=c$, очевидно, не имеет корней на $F$ и на $\Delta \backslash(a, b)$. Очевидно также, что при $c \in(0,1)$ оно имеет $n+1 \geqslant k$ корней на $(\alpha, \beta)$. И, наконец, при $c \notin(0,1)$ уравнение $\varphi(x)=c$ имеет единственньй корень на $\Delta \backslash(\alpha, \beta)$. Лемма доказана.

ЗАмечАниЕ 6 . Лемма 16 указывает на неулучшаемость теоремы 4 в следующем смысле: каков бы ни был интервал $(\alpha, \beta)$, содержащий точки множества $E$ из теоремы 4 , но не пересекаюшийся с его ядром Бэра, сушествует система $\Phi=\left\{\varphi_{1}, \varphi_{2}\right\}$ непрерьвных на некотором отрезке $[a, b] \supset E$ функций, $(\alpha, \beta) \subset[a, b]$, чебьшёвская на $E$ (а, по теореме 4 , и на $E \cup(B \backslash N)$ ), но не являюшаяся таковой на $(\alpha, \beta) \backslash N, N=N(\Phi, \Delta, E)$. Действительно, пусть $F$ - порция множества $E$ на $(\alpha, \beta), \varphi_{1}(x) \equiv 1, \varphi_{2}(x) \equiv \varphi(x)$, где $\varphi(x)$ - функция из леммы 16 . Тогда нетрудно увидеть, что множество $(\alpha, \beta) \cap N$ совпадает с полньм прообразом множества $\varphi(F)$, и что на дополнении $(\alpha, \beta) \backslash N$ к нему любой полином вида $c-\varphi(x)$, где $c \in(0,1) \backslash \varphi(F)$, имеет не менее $k$ нулей.

СлеДСТВИЕ 2. Каково бъ ни бъло всюду плотное на $(\alpha, \beta)$ множество $F$ первой категории существует система $\Phi=\left\{\varphi_{1}, \varphi_{2}\right\}$ непрерьвных на $J=[\alpha, \beta]$ функиий, чебьшёвская на $F$, и такая, что

а) ее хааровский аннулятор $N:=N(\Phi, J, F)$ - это всюду плотное на $J$ множество (первой категории),

b) каково бъи ни било множество $E \supset F$ со свойством Бәра, на котором система $\Phi$ является чебъиёвской, разность $J \backslash(E \cup N)$-множество второй категории (т.е. не первой категории).

Действительно, положим $\Phi=\{1, \varphi(x)\}, x \in J$, где $\varphi(x)$ - функция из леммы 16. Тогда аннулятор $N=N(\Phi, J, F)$ является полным прообразом множества $\varphi(F)$, так что $N$ - множество первой категории, плотное на $J$. Если $E-$ произвольное множество со свойством Бэра, содержащее $F$, и на $E$ система $\Phi$ является чебышёвской, то по теореме $4 \Phi$ является чебьшёвской на $E \cup(B \backslash N)$, где $B$ - ядро Бэра множества $E$. Если бы $B$ совпало с $(\alpha, \beta)$, то система $\Phi$ была бы чебышёвской на $(\alpha, \beta) \backslash N$, что не верно. Поэтому множество $J \backslash E$ не может быть первой категории.

Заметим еще, что из леммы 16 без труда получается также такое следствие: каким бы ни было всюду плотное на отрезке $J=[\alpha, \beta]$ множество $F$ первой категории ( $F$ - может иметь при этом меру $\beta-\alpha$ ) и положительное $\mu<\beta-\alpha$ сушествует подпространство $L \subset C(J), \operatorname{dim} L=2$, чебышёвское на $F$ и не являющееся таковым на любом интервале $\left(\alpha^{\prime}, \beta^{\prime}\right) \subset J$ длины $\beta^{\prime}-\alpha^{\prime} \geqslant \mu$. (Надо лишь в доказательстве леммы 16 точки $x_{1}, x_{2}, \ldots, x_{n}$ взять так, чтобы вьполнялись неравенства $\left.x_{i+1}-x_{i}<\mu, i=0,1, \ldots, n\right)$. 
Таким образом, в общем случае для существования чебышёвского продолжения системы функций, чебышёвской на множестве $E$, с $E$ до некоторых естественных границ необходимо, чтобы множество $E$ было в топологическом смысле достаточно массивным.

\section{§ 3. Обобщения и аналоги теоремы Хаара}

Теорема Хаара дает исчерпьвающий признак тех линейных конечномерных подпространств $L \subset C(E)$, которые с весом $p(x) \equiv(1,1)$ составляют пару $(p, L)$, являющуюся системой единственности на $C(E), E$-компакт. В обшем случае возникает задача описать веса $p$, при которых этот замечательный по простоте признак сохраняется для пары $(p, L)$ в виде, достаточно близком к классическому. В конце $\S 1$ мы видели, что к таким весам можно отнести веса без точек полувырожденности (см. теорему 3). Результаты $\S 2$ позволяют нам теперь продвинуться дальше. При этом проблема состоит, прежде всего, в том, чтобы на основании условия " $(p, L)-$ система единственности", с делать возможно более общие выводы о подмножествах компакта $E$, на которых подпространство $L$ является чебышёвским.

Для дальнейшего отметим следующие свойства носителей и аннуляторов компонент $p_{-}(x)$ и $p_{+}(x)$ полунепрерывного веса. Если неотрицательная функция $q(x)$ полунепрерывна сверху на компакте $E \subset \mathbb{R}$, то ее носитель

$$
M(q, E)=\bigcup_{k}\left\{x \in E: q(x) \geqslant \frac{1}{k}\right\}
$$

представляет собой объединение замкнутых множеств, т.е. $M(q, E)$ - множество типа $F_{\sigma}$, а аннулятор $N(q, E)$ есть множество типа $G_{\delta}$. Поэтому, если $E$ содержит интервал, на котором $N(q, E)$ плотно, то $N(q, E)$ - остаточное множество на таком интервале. Если $\Delta$ - это промежуток, $p=\left(p_{-}, p_{+}\right) \in \mathscr{P}(\Delta)$, то аннулятор $N(p, \Delta)$ веса $p$ является множеством первой категории на $\Delta$ тогда и только тогда, когда $N(p, \Delta)$ нигде не плотно на $\Delta$.

Пусть $G \subset E \subset \mathbb{R}$. Через $\mathscr{H} \mathscr{P}(G)$ обозначим семейство весов $p=\left(p_{-}, p_{+}\right) \in$ $\mathscr{P}(E)$, удовлетворяющих следующим двум условиям:

1) носители $M\left(p_{-}, E\right), M\left(p_{+}, E\right)$ всюду плотны на $G$;

2 ) аннулятор $N(p, E)$ веса $p$ нигде не плотен на $G$.

ОПреДЕЛЕНИЕ 9. Пусть $p \in \mathscr{P}(E)$, где $E$ - компакт на $\mathbb{R}$ с непустьм открытым ядром $E^{0}$. Максимальное открытое подмножество $G$ множества $E$ такое, что $p \in \mathscr{H} \mathscr{P}(G)$, назовем хааровским ядром веса $p$ на $E$ и обозначим через $H(p)=H(p, E)$.

Если $H(p, E)=E^{0}$, то хааровское ядро веса $p$ назовем максимальным.

Очевидно, хааровское ядро $H(p, E)$ является максимальным тогда и только тогда, когда $E^{0}=\Pi^{0}(p, E)$, а аннулятор $N(p, E)$ веса $p$ нигде не плотен на $E^{0}$. Нетрудно увидеть, что хааровское ядро $H(p, E)$ - регулярное открытое множество.

В приводимых ниже леммах 17-19 используются следуюшие обозначения: $\Pi:=$ $\Pi(p, E)$ - ядро веса $p, \Pi^{0}$ - открытое ядро множества $\Pi, \bar{N}$ - замыкание аннулятора $N(p, E),(\bar{N})^{0}$ - открытое ядро множества $\bar{N}, \overline{\left((\bar{N})^{0}\right)}$ - замыкание множества $(\bar{N})^{0}$, $F:=F(p, E),(\bar{F})^{0}$ - открытое ядро замькания $\bar{F}$ множества $F$.

Непосредственно из определения хааровского ядра вытекает следуюшая лемма. 
Лемма 17. Справедливо равенство $H(p, E)=\Pi^{0} \backslash \overline{\left.(\overline{(N})^{0}\right)}$.

Лемма 18. Пусть $G=\Pi^{0} \backslash \bar{N}$. Тогда $F$ плотно на $G$, и при этом $(\bar{F})^{0} \backslash \bar{N}=G$.

ДокАЗАтЕльство. Достаточно показать, что $F$ плотно на любом интервале, на котором плотны носители $M_{-}:=M\left(p_{-}, E\right)$ и $M_{+}:=M\left(p_{+}, E\right)$ и который не содержит точек из $N(p, E)$.

Предположим, что существует интервал $I=(\alpha, \beta)$, не содержащий точек из $F$, из $N$, и на котором $M_{-}$и $M_{+}$плотны. С одной стороны это означает, что множества $A:=I \cap M_{+}$и $B:=I \cap M_{-}$не пересекаются и плотны на $I$. С другой, поскольку $A=I \cap N\left(p_{-}, E\right)$, а $B=I \cap N\left(p_{+}, E\right)$, множества $A$ и $B$ являются множествами типа $G_{\delta}$. Поэтому множества $A$ и $B$ остаточные на $I$ и, следовательно, пересекаются. Противоречие. Лемма доказана.

Лемма 19. Пусть $G=\Pi^{0} \backslash \bar{N}$. Тогда ядро Бәра множества $G \cup F$ совпадают с хааровским ядром $H(p, E)$.

ДокАЗАТЕльСтво. Пусть множество $F \backslash G$ плотно на каком-либо интервале. Тогда в силу равенства $(\bar{F})^{0} \backslash \bar{N}=G$ (см. лемму 18) на этом интервале плотно также $N$, так что $N$ - остаточное множество на этом интервале. Следовательно, множество $F$ на этом интервале - первой категории. С другой стороны, ядро Бэра множества $G$ совпадает с $H(p, E)$ (ср. равенство в лемме 17 с определением $G$ ). Лемма доказана.

Хааровским аннулятором $N(L, E, A)$ конечномерного подпространства $L \subset C(E)$ на $E \backslash A$ назовем хааровский аннулятор $N(\Phi, E, A)$ системы $\Phi$ на $E \backslash A$, где $\Phi=\left\{\varphi_{1}, \ldots, \varphi_{n}\right\}$ - базис в $L$ (см. определение 8 ).

В следуюшей лемме рассматривается взаимное расположение хааровского аннулятора подпространства $L$ и аннулятора веса $p$ в случае, когда пара $(p, L)$ является системой единственности.

ЛЕмма 20. Пусть $E$ - компакт на $\mathbb{R}, L$ - конечномерное подпространство в $C(E), p \in \mathscr{P}(E),(p, L)$ - система единственности на $C(E, p, L)$. Тогда для любого подмножества $A \subset E$, $\operatorname{card} A \geqslant \operatorname{dim} L$, на котором $L$ является чебъиёвским подпространством, имеем $N(L, E, A) \subset N(p, E)$.

ДокаЗАтельство. Пусть $x_{0} \in N(L, E, A)$. Это означает, что сушествуют различные точки $x_{1}, \ldots, x_{n-1} \in A$ и полином $l \in L$ такой, что $l\left(x_{0}\right)=l\left(x_{1}\right)=$ $\cdots=l\left(x_{n-1}\right)=0$. Поэтому, если $\Phi(x)=\left\{\varphi_{1}(x), \ldots, \varphi_{n}(x)\right\}-$ базис в $L$, a $r$ - ранг системы векторов $\left\{\Phi\left(x_{0}\right), \Phi\left(x_{1}\right), \ldots, \Phi\left(x_{n-1}\right)\right\}$, то $r<n$. По лемме 4 на множестве $X=\left\{x_{0}, x_{1}, \ldots, x_{n-1}\right\}$ для каждой однозначной ветви $\sigma(x)$ сигнатуры веса $p$ найдется функция $\varphi(x)=c_{1} \varphi_{1}(x)+\cdots+c_{n} \varphi_{n}(x)$ такая, что

$$
\sigma\left(x_{k}\right) \sum_{i=1}^{n} c_{i} \varphi_{i}\left(x_{k}\right)>0, \quad k=0,1, \ldots, n-1 .
$$

Если допустить, что $x_{0} \notin N(p, E)$, то сигнатура $\sigma(p ; x)$ веса $p$ на $X$ является полным или квазиполным $\sigma$-вектором (см. определение 3 ). По лемме 6 в этом случае $r=\operatorname{card} X$, т.е. $r=n$. Противоречие говорит о том, что $x_{0} \in N(p, E)$. Лемма доказана. 
Следуюшая теорема решает задачу чебышёвского продолжения подпространства $L$ вплоть до точек вырожденности веса $p$ (не включая в это продолжение самих этих точек).

Tеорема 6. Пусть $E$ - компакт на $\mathbb{R}, p \in \mathscr{P}(E), F:=F(p, E), L-\kappa o-$ нечномерное подпространство в $C(E), \operatorname{dim} L \leqslant \operatorname{card} F$. Тогда если $(p, L)-$ система единственности на $C(E)$, то подпространство $L$ является чебишёвским на множсестве $F \cup(H \backslash N)$, где $H$ - хааровское ядро $H(p, E)$ с любой присоединенной $к$ нему его достижсмой граничной точкой, $N=N(p, E)$ аннулятор веса р.

ДокАЗАтЕльство. 1) По лемме 7 подпространство $L$ при каждом $\xi \in M:=$ $M(p, E)$ является чебышёвским на множестве $F \cup\{\xi\}$ и, таким образом, $N(L, M, F)$ $=\varnothing$. Положим $G=\Pi^{0} \backslash \bar{N}$, где $\Pi^{0}$ - открытое ядро множества $\Pi(p, E), \bar{N}-$ замыкание $N(p, E)$. Очевидно, $G \subset M, N(L, G, F)=\varnothing$. По лемме 18 множество $F$ плотно на $G$.

2) Поэтому по теореме 5 подпространство $L$ является чебышёвским на $A:=$ $G \cup F$, а по теореме 4 также и на $A \cup\left(B \backslash N_{1}\right)$, где $B$ - это ядро Бэра множества $A$ с любой присоединенной к нему его достижимой граничной точкой, $N_{1}:=N(L, B \cup A, A)$. По лемме 19 ядро Бэра множества $A$ совпадает с $H(p, E)$, так что будем считать $B=H$. Так как $B \cup A \subset E$, то $N_{1} \subset N(L, E, A)$, а по лемме $20 N(L, E, A) \subset N$, откуда $N_{1} \subset N$. В итоге получаем

$$
A \cup\left(B \backslash N_{1}\right)=(G \cup F) \cup\left(H \backslash N_{1}\right)=F \cup\left(H \backslash N_{1}\right) \supset F \cup(H \backslash N) .
$$

Теорема доказана.

СлЕДСТВИЕ 3. Пусть $\Delta=[a, b], p \in \mathscr{P}(\Delta), H(p, \Delta)=(a, b), L$ - конечномерное подпространство в $C(\Delta)$. Тогда если $(p, L)$ - система единственности на $C(\Delta)$, то подпространство $L$ является чебишёвским на $[a, b) \backslash N(p, \Delta)$ и $(a, b] \backslash N(p, \Delta)$.

ЗАмечАниЕ 7. То, что здесь не может быть гарантирована чебышёвость подпространства $L$ на $[a, b] \backslash N(p, \Delta)$ (в том числе и при пустом аннуляторе $N(p, \Delta)$ ) демонстрируют пары $\left(p, L_{2}\right)$ и $\left(q, L_{1}\right)$ из примера $2(\S 1)$.

Из теоремы 6 и лемм 2 и 8 непосредственно получим следующее утверждение, в котором $\partial E$, как обычно, обозначает границу множества $E$.

Tеорема 7. Пусть $E$ - компакт на $\mathbb{R}, p \in \mathscr{P}(E), E^{0}=H(p, E), x^{*}-\kappa a-$ кая-либо достижимая граничная точка множества $E^{0}, \partial E \backslash\left\{x^{*}\right\} \subset F(p, E)$, $L$ - конечномерное подпространство в $C(E), \operatorname{dim} L \leqslant \operatorname{card} F(p, E)$. Тогда для того чтобъ пара $(p, L)$ была системой единственности на $C(E)$ необходимо и достаточно, чтобъ подпространство $L$ было чебышёвским на $M(p, E), \boldsymbol{в}$ частности, если $N(p, E)=\varnothing$, то на всем множестве $E$.

Всюду ниже $a \vee b \in F$ обозначает, как обычно, что хотя бы одна из точек $a$ или $b$ принадлежит $F$.

СлЕДСТВИЕ 4. Пусть $\Delta=[a, b], p \in \mathscr{P}(\Delta), H(p, \Delta)=(a, b), a \vee b \in F(p, \Delta)$, $L$ - конечномерное подпространство в $C(\Delta)$. Тогда для того чтобъ $(p, L)$ была системой единственности на $C(\Delta)$ необходимо и достаточно, чтобъ $L$ бъло чебъиёвским на $M(p, \Delta)$. 
ЗАмечаниЕ 8. Рассматривая приближения непрерывных функций на компак$m e E$ элементами подпространства $L \subset C(E)$, можно считать, по сушеству не ограничивая общности, что открытое ядро $N^{0}$ аннулятора $N(p, E)$ веса $p$ пусто. Действительно, если $N^{0} \neq \varnothing$ и подпространство $L$ не вырождается на $E^{*}=E \backslash N^{0}$ (т.е. если линейная размерность сужения $\left.L\right|_{E^{*}}$ подпространства $L$ на $E^{*}$ равна $\operatorname{dim} L$ ), то интересующие нас вопросы единственности элемента наилучшего приближения можно решать, приближая непрерывные функции на компакте $E^{*}$ элементами подпространства $\left.L\right|_{E^{*}}$. Если $N^{0}=\varnothing$, то замыкание $\bar{M}(p, E)=\operatorname{supp} p$ носителя $M(p, E)$ совпадает с $E$, и подпространство $L$ никогда не вырождается на $M(p, E)$. Это и будет предполагаться в дальнейшем.

Поставим теперь задачу получения чебышёвского продолжения подпространства $L$, включающего в себя также точки вырожденности веса $p$, т.е. точки аннулятора $N(p, E)$. Заметим, что эти точки на компакте $E$ могут составлять множество положительной меры (даже при условии, что открытое ядро множества $N(p, E)$ пусто). Простые примеры показывают, что ситуация здесь может быть существенно различной в зависимости от того, является ли компакт $E$ связным множеством или нет. Действительно, пусть $E=[-1,1] \cup\{2\}, L=\left\{c_{1}+c_{2} x(x-2)\right\}$, $p_{-}(x), p_{+}(x)$ - любые полунепрерывные сверху, положительные на $E \backslash\{0\}$ функции, $p_{-}(0)=p_{+}(0)=0$. Тогда на носителе $M(p, E)=E \backslash\{0\}$ подпространство $L$ чебьшёвское, $(p, L)$ - система единственности на $C(E), N(L, E)=\varnothing$, но на $E$ подпространство $L$ чебышёвским не является. Таким образом, чебышёвского продолжения с носителя $M(p, E)$ на $E$ здесь не существует, хотя "особенностью" (обусловленной вырожденностью веса $p$ ) является единственная внутренняя точка множества $E$ (при пустом аннуляторе $N(L, E)$ подпространства $L$ ). В то же время отметим, что $L$ является чебышёвским подпространством на множестве $[-1,1]=E \backslash\{2\}$, содержашем внутри себя $N(p, E)(\neq \varnothing)$.

Заметим также, что в этом примере $N(p, E)$ совпадает с хааровским аннулятором $N(L, E, F)$, где $F=F(p, E)=M(p, E)$. Очевидно, одно это определяет невозможность чебышёвского продолжения, включаюшего точки из $N(p, E)$. Это, наряду с теоремой 4 , позволяет предположить, что в решении поставленной задачи на произвольном компакте $E$ существенную роль должна сыграть такая характеристика, как хааровский аннулятор $N(L, E, F)$, с тем или иньм множеством $F$, на котором тем или иным образом (на основании того, что $(p, L)$ - система единственности) уже установлена чебышёвость подпространства $L$. Однако ниже мы отдаем предпочтение формулировкам, в которых участвует лишь обычный аннулятор $N(L, E):=\{x \in E: l(x)=0 \quad \forall l \in L\}$ подпространства $L$ на $E$ - характеристика значительно более прозрачная. За хааровским аннулятором сохраняется роль промежуточной характеристики, полезной при доказательствах.

Следующая теорема показьвает также, что хааровское ядро $H(p, E)$ - это максимальное открытое множество для данного веса $p$, на котором для любого приближающего конечномерного подпространства $L$ выполняется необходимое условие Хаара единственности элемента наилучшего приближения.

ТеОрема 8. 1) Пусть $E$ - компакт на $\mathbb{R}$ с непустым открытым ядром $E^{0}$, $L$ - конечномерное подпространство в $C(E), p \in \mathscr{P}(E), H$ - хааровское ядро $H(p, E)$ любой присоединенной $\kappa$ нему его достижимой граничной точкой, $H(p, E) \neq \varnothing$. Тогда если $(p, L)$ - система единственности на $C(E)$, то подпространство $L$ является чебышёвским на $H \backslash N(L, E)$, в частности, при 
$N(L, E)=\varnothing$ на всем множестве $H$.

2) При этом если $H(p, E) \neq E^{0}$ и $\widetilde{G}-$ произвольное открытое подмножество множества $E^{0}$, содержащее $H(p, E)$ в качестве собственного подмножества, то существует такое подпространство $L_{0} \subset C(E), \operatorname{dim} L_{0} \geqslant 2$, $N\left(L_{0}, E\right)=\varnothing$, что $\left(p, L_{0}\right)$ является системой единственности на $C(E)$, но $L_{0}$ не является чебышёвским подпространством на $\widetilde{G}$.

ДокАЗАТЕльство. 1) Первая часть теоремы фактически установлена выше при доказательстве теоремы 6 . Надо лишь в п. 2) этого доказательства под $F$ понимать ту часть остова $F=F(p, E)$ веса $p$, которая лежит на $G$. Тогда все, что есть в п. 2) остается в силе, но зато теперь по лемме $12 N_{1}=N(L, B)$, так что $L$ является чебьшёвским на множестве $A \cup\left(B \backslash N_{1}\right)=H \backslash N(L, B)=H \backslash N(L, E)$. (Предостережем от ошибки считать, что в обшем случае $N_{1}:=N(L, B \cup A, A) \subset N(L, B, G)$ - см. замечание 5.)

2) Доказательству второй части теоремы предпошлем две леммы.

Лемма 21. Пусть $n$ - натуральное число, $\Psi=\left\{\psi_{1}, \ldots, \psi_{n}\right\}$ - система Чебышёва на $\Delta=[a, b],(\alpha, \beta) \subset(a, b), \Phi=\left\{\varphi_{1}, \ldots, \varphi_{n}\right\}$ - система непрерывных на $\Delta$ функций $\varphi_{i}$ таких, что $\varphi_{i}(x)=\psi_{i}(x)$ при $x \in \Delta \backslash(\alpha, \beta)$ и $\varphi_{i}(x)$ линейна на $[\alpha, \beta], i=1, \ldots, n, L$ - линейное подпространство в $C(\Delta)$ с базисом $\Phi$, $p=\left(p_{-}, p_{+}\right)-$вес из $\mathscr{P}(\Delta)$ такой, что на $[\alpha, \beta]$ либо функиия $p_{-}(x)$, либо $p_{+}(x)$ тождественна нулю, в остальном вес р произвольный. Тогда если $C(\Delta, p, L)$ непусто, то $(p, L)$ - система единственности на $C(\Delta, p, L)$. При $n \geqslant 3$ подпространство $L$ не является чебышёвским на $\Delta$.

ДокАЗАТЕЛЬСтво. Поскольку каждая функция из $L$ линейна на $[\alpha, \beta]$, то для полинома $l(x)=a_{1} \varphi_{1}(x)+\cdots+a_{n} \varphi_{n}(x)$ могут представиться только следующие три возможности:

1) $l(x) \neq 0$ при $x \in(\alpha, \beta)$;

2) $l(x)=0$ в единственной точке $x_{1}$ интервала $(\alpha, \beta)$;

3) $l(x) \equiv 0$ на $(\alpha, \beta)$,

В случае 1) все нули $l(x)$ лежат на множестве $\Delta \backslash(\alpha, \beta)$, на котором система $\Phi$ совпадает с системой Чебьшёва $\Psi$. Поэтому всегда сушествует полином $c_{1} \psi_{1}(x)+\cdots+c_{n} \psi_{n}(x)$, принимающий в этих нулях наперед заданные значения. Тем же свойством в случае 1 ) обладает соответствующий ему полином $f(x)=c_{1} \varphi_{1}(x)+\cdots+c_{n} \varphi_{n}(x)$ из $L$.

В случае 2) полином $g(x)=a_{1} \psi_{1}(x)+\cdots+a_{n} \psi_{n}(x)$, совпадающий с $l(x)=$ $a_{1} \varphi_{1}(x)+\cdots+a_{n} \varphi_{n}(x)$ на $\Delta \backslash(\alpha, \beta)$, принимает в точках $\alpha$ и $\beta$ значения разных знаков и имеет, таким образом, на $(\alpha, \beta)$ по крайней мере один нуль. Поэтому на $\Delta \backslash[\alpha, \beta]$ полином $l(x)$, как и $g(x)$, имеет не более $n-2$ нулей. Добавим к этим нулям точки $\alpha$ и $\beta$. Полученное множество $X_{0}$ состоит не более чем из $n$ точек и содержится в множестве $\Delta \backslash(\alpha, \beta)$, на котором $\Phi$, совпадая с $\Psi$, является системой Чебышёва. Поэтому сушествует полином $f(x) \in L$, принимаюший в точках множества $X_{0}$ любые значения, в частности, в точках $\alpha$ и $\beta$ положительные значения, если $p_{+}(x) \equiv 0$ на $[\alpha, \beta]$, и отрицательные значения, если $p_{-}(x) \equiv 0$ на $[\alpha, \beta]$. Следовательно, значения соответствуюшего знака функция $f(x)$ как функция, линейная на $[\alpha, \beta]$, принимает также в нуле $x_{1} \in(\alpha, \beta)$ полинома $l(x)$.

В случае 3$)$ множество $X$ нулей полинома $l(x)$ состоит из отрезка $[\alpha, \beta]$ и еше не более чем из $n-3$ нулей, лежащих на $\Delta \backslash[\alpha, \beta]$. Присоединим к этим $(n-3)$-ем нулям 
точки $\alpha$ и $\beta$. Полученное множество $X_{0}$ состоит не более чем из $n-1$ точек и лежит на множестве $\Delta \backslash(\alpha, \beta)$. Поэтому существует полином $f \in L$, принимающий в точках множества $X_{0}$ любые наперед заданные значения. Потребуем, в частности, чтобы в точках $\alpha$ и $\beta$, а следовательно, и всюду на $[\alpha, \beta]$ он принимал значения того знака, который является индексом компоненты веса $p=\left(p_{-}, p_{+}\right)$, тождественной нулю на $[\alpha, \beta]$.

Итак, во всех трех случаях выполняются условия леммы 5 , при которых $(p, L)$ является системой единственности на $C(\Delta, p, L)$. Очевидно, что при $n \geqslant 3$ множество полиномов из $L$, тождественных нулю на $[\alpha, \beta]$, образует подпространство размерности $n-2$. Лемма доказана.

Лемма 22. Пусть $a<\alpha<\beta<b, \gamma=(\beta-\alpha) / 4, \psi(x)=x\left(1-x^{2}\right), \varphi(x)=$ $\psi((x-(\alpha+\beta) / 2) / \gamma), L=\left\{c_{1}+c_{2} \varphi(x): c_{1}, c_{2} \in \mathbb{R}\right\}, x \in \Delta=[a, b], p=\left(p_{-}, p_{+}\right)$ - полунепрерывный сверху на $\Delta$ вес такой, что на $(\alpha, \beta)$ либо функция $p_{-}(x)$, либо $p_{+}(x)$ тождественна нулю, в остальном вес произвольный. Тогда если $C(\Delta, p, L)$ непусто, то $(p, L)$ - система единственности на $C(\Delta, p, L)$. При этом подпространство $L$ не является чебышёвским на $\Delta$.

ДокАЗАТЕЛЬСтво. Поскольку уравнение $\psi(x)=c=$ const вне отрезка $[-2,2]$ имеет не более одного корня и при этом (если корень есть) не имеет корней на $[-2,2]$, то это же свойство вьполняется и для уравнения $\varphi(x)=c$ и отрезка $(\alpha, \beta)$. Поэтому, пользуясь леммой 5 , заключаем, что если $C(\Delta, p, L) \neq \varnothing$, то $(p, L)-$ система единственности. Лемма доказана.

Продолжим доказательство теоремы 8 . Поскольку $H(p, E)$ - регулярное открытое множество, то сушествует непустой интервал $(c, d) \subset \widetilde{G} \backslash H(p, E)$. По определению хааровского ядра на $(c, d)$ найдется такой подинтервал $(\alpha, \beta)$, что либо а) одна из функций $p_{-}(x), p_{+}(x)$ тождественна нулю на $(\alpha, \beta)$, либо b) оба множества $N\left(p_{-}, E\right), N\left(p_{+}, E\right)$ плотны на $(\alpha, \beta)$ (и, тем самьм, являются на нем остаточными). Будем считать функции $p_{-}(x)$ и $p_{+}(x)$ продолженными нулем вне $E$, и пусть $\Delta-$ это любой отрезок, содержаший $E$.

В случае а) согласно леммам 21 и 22 , а также лемме 2 при каждом $n \geqslant 2$ найдется подпространство $L_{0} \subset C(E)$ такое, что $\operatorname{dim} L_{0}=n, N\left(L_{0}, E\right)=\varnothing,\left(p, L_{0}\right)$ система единственности на $C(E)$, но подпространство $L_{0}$ не является чебьшёвским на $(\alpha, \beta)$.

В случае $\mathrm{b})$, очевидно, можно считать, что на $(\alpha, \beta)$ каждый из носителей $M\left(p_{-}, E\right), M\left(p_{+}, E\right)$ также плотен на $(\alpha, \beta)$. Множество

$$
F:=\left(M\left(p_{-}, E\right) \cap M\left(p_{+}, E\right)\right) \cap(\alpha, \beta)
$$

в этом случае является множеством первой категории на $(\alpha, \beta)$. Рассмотрим систему $\{1, \varphi(x)\}, x \in \Delta$, где $\varphi(x)$ - функция, определенная в лемме 16 . Порождаемое ею пространство $L_{0}$ вместе с весом $p$ образует пару $\left(p, L_{0}\right)$, являюшуюся системой единственности на $C(E)$. Действительно, так как $M(p, E) \subset F \cup(\Delta \backslash(\alpha, \beta))$, то по лемме 16 на $M(p, E)$ любая функция из $L_{0} \backslash\{0\}$ имеет не более одного нуля. Поэтому очевидным образом выполняются условия леммы 5 , так что $(p, L)$ - система единственности на $C(E, p, L)$, а следовательно, и на $C(E)$ (лемма 2$)$. В то же время указанное подпространство $L_{0}$ не является чебышёвским на $\widetilde{G}(\varphi(x)$ имеет на $(\alpha, \beta)$ не менее $k \geqslant 3$ нулей). Теорема 8 доказана. 
СлеДСТвИЕ 5. Пусть $\Delta=[a, b], p \in \mathscr{P}(\Delta), H(p, \Delta)=(a, b), L$ - конечномерное подпространство в $C(\Delta)$. Тогда если $(p, L)$ - система единственности на $C(\Delta)$, то подпространство $L$ является чебьиёвским на $[a, b) \backslash N(L, \Delta)$ и на $(a, b] \backslash N(L, \Delta)$. Если при этом $N(L, \Delta)=\varnothing$, то $L$ является чебышёвским на каждом из промежутков $[a, b) u(a, b]$.

ОПРЕДЕЛЕНИЕ 10. Будем говорить, что вес $p(x)=\left(p_{-}(x), p_{+}(x)\right)$ в точках $a$ и $b$ одинаково полувырожден, если либо $p_{-}(a)=p_{-}(b)=0, p_{+}(a) p_{+}(b)>0$, либо $p_{+}(a)=p_{+}(b)=0, p_{-}(a) p_{-}(b)>0$. Если же либо $p_{-}(a)=p_{+}(b)=0$, $p_{+}(a) p_{-}(b)>0$, либо $p_{+}(a)=p_{-}(b)=0, p_{-}(a) p_{+}(b)>0$, то будем говорить, что вес $p$ в точках $a$ и $b$ противоположно полувырожден.

Tеорема 9. Пусть $\Delta=[a, b], L$ - конечномерное подпространство в $C(\Delta)$, $N(L, \Delta)=\varnothing, p \in \mathscr{P}(\Delta), H(p, \Delta)=(a, b)$, и пусть при нечетной размерности $\operatorname{dim} L$ вес р в точках а и $b$ одинаково полувырожден, а при четной - противоположно полувырожден. Тогда для того чтобы пара $(p, L)$ была системой единственности на $C(\Delta)$ необходимо и достаточно, чтобъ подпространство $L$ бъло чебшиёвским на каждом из промежстков $[a, b)$ u $(a, b]$.

ДокАзАТЕЛЬСтво. Необходимость уже установлена - см. следствие 5.

Достаточность. Поскольку $L$ является чебышёвским на $[a, b)$ и $(a, b]$, то согласно лемме 5 достаточно доказать следуюшее утверждение.

Лемма 23. Пусть $L$ - п-мерное чебъшёвское подпространство на каждом из промежутков $[a, b)$ и $(a, b], a=x_{1}<x_{2}<\cdots<x_{n-1}<x_{n}=b$, $\sigma=\left(\sigma_{1}, \ldots, \sigma_{n}\right)$, где $\sigma_{i}$ принимает либо значение 1 , либо -1, причем $\sigma_{1} \sigma_{n}=1$ при нечетном $n$ и $\sigma_{1} \sigma_{n}=-1$ при четном $n$. Тогда каким бы ни был вектор $\sigma$ с наложенными на него выше условиями существует полином $l \in L$ такой, что $\operatorname{sign} l\left(x_{i}\right)=\sigma_{i}$ при всех $i=1, \ldots, n$.

ДокаЗАТЕЛЬСтво. Пусть $\Phi(x)=\left(\varphi_{1}(x), \ldots, \varphi_{n}(x)\right), x \in \Delta=[a, b],-$ базис в $L$. Требуется доказать совместность системы неравенств

$$
\sigma_{i} \sum_{k=1}^{n} c_{k} \varphi_{k}\left(x_{i}\right)>0, \quad i=1, \ldots, n,
$$

относительно неизвестных $c_{1}, \ldots, c_{n}$. Предположим, что система несовместна. Тогда (см., например, $\left[18\right.$, с. 216]) существуют числа $\lambda_{i} \geqslant 0, i=1, \ldots, n$, не все равные нулю, такие, что

$$
\sum_{i=1}^{n} \lambda_{i} \sigma_{i} \varphi_{k}\left(x_{i}\right)=0, \quad k=1, \ldots, n
$$

Рассмотрим определитель, $x \in \Delta$,

$$
\left|\begin{array}{ccc}
\sum_{i=1}^{n} \lambda_{i} \sigma_{i} \varphi_{1}\left(x_{i}\right) & \ldots & \sum_{i=1}^{n} \lambda_{i} \sigma_{i} \varphi_{n}\left(x_{i}\right) \\
\varphi_{1}(x) & \ldots & \varphi_{n}(x) \\
\varphi_{1}\left(x_{2}\right) & \ldots & \varphi_{n}\left(x_{2}\right) \\
\ldots \ldots \ldots \ldots \ldots \ldots \ldots \ldots \ldots \ldots \ldots \ldots \ldots \ldots \ldots \ldots \ldots \ldots & \ldots \ldots \ldots
\end{array}\right| .
$$


Очевидно, он равен нулю. Представляя (12) в виде суммы $n$ определителей, первые строки которых в сумме дают первую строку определителя (12), и отбрасывая определители с двумя одинаковыми строками, получим

$$
\lambda_{1} \sigma_{1}\left|\begin{array}{ccc}
\varphi_{1}\left(x_{1}\right) & \ldots & \varphi_{n}\left(x_{1}\right) \\
\varphi_{1}(x) & \ldots & \varphi_{n}(x) \\
\varphi_{1}\left(x_{2}\right) & \ldots & \varphi_{n}\left(x_{2}\right) \\
\ldots \ldots \ldots \ldots \ldots & \ldots \ldots \\
\varphi_{1}\left(x_{n-1}\right) & \ldots & \varphi_{n}\left(x_{n-1}\right)
\end{array}\right|+\lambda_{n} \sigma_{n}\left|\begin{array}{ccc}
\varphi_{1}\left(x_{n}\right) & \ldots & \varphi_{n}\left(x_{n}\right) \\
\varphi_{1}(x) & \ldots & \varphi_{n}(x) \\
\varphi_{1}\left(x_{2}\right) & \ldots & \varphi_{n}\left(x_{2}\right) \\
\ldots \ldots \ldots \ldots \ldots & \ldots \ldots \ldots \\
\varphi_{1}\left(x_{n-1}\right) & \ldots & \varphi_{n}\left(x_{n-1}\right)
\end{array}\right|=0
$$

Введем обозначение

$$
D\left(y_{1}, y_{2}, \ldots, y_{n}\right):=\left|\begin{array}{ccc}
\varphi_{1}\left(y_{1}\right) & \ldots & \varphi_{n}\left(y_{1}\right) \\
\varphi_{1}\left(y_{2}\right) & \ldots & \varphi_{n}\left(y_{2}\right) \\
\ldots \ldots \ldots \ldots & \ldots \\
\varphi_{1}\left(y_{n}\right) & \ldots & \varphi_{n}\left(y_{n}\right)
\end{array}\right|
$$

Если во втором определителе в (13) первую строку поставить на последнее место, то (13) перепишется в виде

$$
\lambda_{1} \sigma_{1} D\left(x_{1}, x, x_{2}, \ldots, x_{n-1}\right)+\lambda_{n} \sigma_{n} D\left(x, x_{2}, \ldots, x_{n}\right)(-1)^{n-1}=0 .
$$

Заметим, что при $x_{1}<x<x_{2}$ знаки определителей $D\left(x_{1}, x, x_{2}, \ldots, x_{n-1}\right)$ и $D\left(x, x_{2}, \ldots, x_{n}\right)$ совпадают. Действительно, из чебьшёвости системы $\Phi(x)$ на $[a, b)$ следует, что определитель $D\left(x_{1}, x, x_{2}, \ldots, x_{n-1}\right), x_{1}<x<x_{2}$, отличен от нуля и что при всевозможных изменениях положения точек $x_{1}, x, x_{2}, \ldots, x_{n-1}$, $x_{1}<x<x_{2}<\cdots<x_{n-1}$, не меняющих порядка их следования на промежутке $[a, b)$, знак этого определителя не меняется. То же относится и к определителю $D\left(x, x_{2}, \ldots, x_{n}\right)$ на $(a, b]$. Поэтому оба эти определителя имеют тот же знак, что и определитель $D\left(y_{1}, y_{2}, \ldots, y_{n}\right)$, где $y_{i}$ - произвольно взятые точки такие, что $a<y_{1}<y_{2}<\cdots<y_{n}<b$.

Поскольку $\Phi(x)=\left\{\varphi_{1}(x), \ldots, \varphi_{n}(x)\right\}$ - система Чебышёва на $(a, b)$, то ранг системы векторов $\Phi\left(x_{2}\right), \ldots, \Phi\left(x_{n-1}\right)$ равен $n-2$, и система уравнений

$$
\sum_{i=2}^{n-1} \lambda_{i} \sigma_{i} \varphi_{k}\left(x_{i}\right)=0, \quad k=1, \ldots, n
$$

относительно неизвестных $\lambda_{i} \sigma_{i}, i=2, \ldots, n-1$, имеет лиш нулевое решение. Так как не все $\lambda_{i}$ равны нулю, то отсюда и из (14) получим, что $\lambda_{1}>0, \lambda_{n}>0$. Итак, величины $\lambda_{1} D\left(x_{1}, x, x_{2}, \ldots, x_{n-1}\right)$ и $\lambda_{n} D\left(x, x_{2}, \ldots, x_{n}\right)$ в равенстве (14) одного знака, откуда следует, что $\sigma_{1}$ и $\sigma_{n}$ одного знака при четном $n$ и разных знаков при нечетном $n$. Это противоречит условию леммы. Лемма доказана.

Доказательство теоремы 9 также завершено. 
ЗАмечание 9 . Полином $l$ в лемме 23 не может быть получен посредством интерполяции в точках $x_{1}=a, x_{2}, \ldots, x_{n-1}, x_{n}=b$, поскольку при отсутствии чебышёвости $L$ на всем отрезке $[a, b]$ имеется, вообше говоря, определенная связь значений искомого полинома $l$ в этих точках. Например, пусть $L=\left\{c_{1} \varphi_{1}+c_{2} \varphi_{2}\right\}$, где $\varphi_{1}(x)=x, \varphi_{2}(x)=1-x^{2},[a, b]=[-1,1], x_{1}=-1, x_{2}=1, \sigma=(1,-1)$. Тогда если $l \in L, \operatorname{sign} l(-1)=1, \operatorname{sign} l(1)=-1$, то $l(-1)=-l(1)$.

В заключение приведем две теоремы, даюшие описание весов $p \in \mathscr{P}(E)$, при которых классическая формулировка теоремы Хаара либо сохраняется полностью (теорема 10), либо сохраняется в несколько более общей форме (теорема 11). Введем необходимые обозначения и определения. Пусть $E$ - компакт; $p \in \mathscr{P}(E) ; \mathscr{Z}(E)$ - множество всех конечномерных подпространств в $C(E) ; \mathscr{Z} T(E)$ - множество всех подпространств $L \in \mathscr{Z}(E)$, чебышёвских на $E$ (см. определение 7$) ; \mathscr{Z}(p, E)$ - множество всех подпространств $L \in \mathscr{Z}(E)$ таких, что $(p, L)$ - система единственности на $C(E) ; \mathscr{L} *(p, E)$ - множество всех подпространств $L \in \mathscr{Z}(E)$ с пустым аннулятором $N(L, E)$ и таких, что $(p, L)$ - система единственности на $C(E)$.

Очевидно, $\mathscr{Z}^{*}(p, E) \subset \mathscr{Z}(p, E)$. Согласно лемме 2 , если $L \in \mathscr{Z}(p, E)$ (или $\left.L \in \mathscr{Z}^{*}(p, E)\right)$, то $\operatorname{dim} L \leqslant \operatorname{card} \Pi(p, E)$. Отметим также, что если $\operatorname{card} \Pi(p, E)=\infty$, то $\mathscr{Z} T(E) \subset \mathscr{Z}^{*}(p, E) \subset \mathscr{Z}(p, E)$ (см. леммы 2 и 8$) ;$ кроме того, если $N(p, E)=\varnothing$, то $\mathscr{Z} *(p, E)=\mathscr{Z}(p, E)$ (см. лемму 20).

ОПРЕДЕЛЕНИЕ 11. Будем назьвать вес $p \in \mathscr{P}(E)$ хааровским (обобщенно-хааровским) на компакте $E$, состоящем из бесконечного числа точек, если $\mathscr{Z} T(E)=$ $\mathscr{Z}(p, E)$, соответственно, $\mathscr{Z} T(E)=\mathscr{Z} *(p, E)$.

Отметим, что если $p$ - хааровский (обобщенно-хааровский) вес на компакте $E$, $\operatorname{card} E=\infty$, то $\operatorname{card} \Pi(p, E)=\infty$ (см. лемму 2).

TEорема 10. Для того чтобы вес $p \in \mathscr{P}(\Delta)$ был хааровским на отрезке $\Delta=[a, b]$ необходимо и достаточно, чтобь одновременно выполнялись следующие условия:

1) $H(p, \Delta)=(a, b)$;

2) $N(p, \Delta)=\varnothing$;

3) $a \vee b \in F(p, \Delta)$.

ДоказАТеЛЬСтво. Необходимость. 1) Пусть $H(p, \Delta) \neq(a, b)$. Тогда по второй части теоремы $8 \mathscr{Z}(p, \Delta) \neq \mathscr{Z} T(\Delta)$, что противоречит условию теоремы $(\mathscr{Z}(p, \Delta)=\mathscr{Z} T(\Delta))$.

2) Пусть $N(p, \Delta) \neq \varnothing$, так что при некотором $x_{0} \in \Delta$ имеем $p_{-}\left(x_{0}\right)=$ $p_{+}\left(x_{0}\right)=0$. Пусть $L^{*} \in \mathscr{Z} T(\Delta), \alpha(x)$ - непрерьвная на $\Delta$ функция такая, что $\alpha\left(x_{0}\right)=0, \alpha(x) \neq 0$ при $x \neq x_{0}$. Тогда $L_{\alpha}:=\left\{l(x) \alpha(x): l \in L^{*}\right\} \in \mathscr{Z}(p, \Delta)$ (см. лемму 8). В то же время, очевидно, $L_{\alpha} \notin \mathscr{Z} T(\Delta)$, что противоречит условию теоремы.

3) Пусть условие $a \vee b \in F(p, \Delta)$ не вьполняется. Тогда по уже доказанному точки $a$ и $b$ являются точками полувырожденности. Пусть $L_{1}-$ произвольное чебышёвское на $[a, b)$ подпространство непрерывных на $[a, b]$ функций, принимающих в точках $a$ и $b$ равные значения (так что $\operatorname{dim} L-$ нечетное число, а $L$ не является чебьшёвским на $[a, b]), L_{2}$ - любое подпространство непрерывных на $\Delta$ функций четной размерности $\operatorname{dim} L_{2}$, чебышёвское на каждом из промежутков $[a, b)$ и $(a, b]$ 
и не являюшееся чебышёвским на $[a, b]$ (например, $L_{2}=\left\{c_{1} \varphi_{1}+c_{2} \varphi_{2}: c_{1}, c_{2} \in \mathbb{R}\right\}$, где $\left.\varphi_{1}(x)=x-(a+b) / 2, \varphi_{2}(x)=(a-x)(b-x)\right)$.

Если в точках $a$ и $b$ вес $p$ одинаково полувырожден, то возьмем пару $\left(p, L_{1}\right)$, a если противоположно полувырожден, то - пару $\left(p, L_{2}\right)$. Эти пары по теореме 9 являются системами единственности на $C(\Delta)$, так что либо подпространство $L_{1}$, либо подпространство $L_{2}$ принадлежит $\mathscr{Z}(p, \Delta)$. В то же время ни $L_{1}$, ни $L_{2}$ не принадлежит $\mathscr{Z} T(\Delta)$, что противоречит условию теоремы.

Достаточность. 1) Поскольку $H(p, \Delta)=(a, b), N(p, \Delta)=\varnothing$, то по следствию 3 если подпространство $L$ принадлежит $\mathscr{Z}(p, \Delta)$, то $L$ является чебышёвским на промежутках $[a, b)$ и $(a, b]$. Если $\operatorname{dim} L=1$, то очевидно, что $L$ является чебьшёвским на $[a, b]$.

2) Предположим, что найдется такое подпространство $L_{0}, \operatorname{dim} L_{0} \geqslant 2$, что $L_{0} \in$ $\mathscr{Z}(p, \Delta)$, но $L_{0} \notin \mathscr{Z} T(\Delta)$. Тогда сушествует такой полином $l_{0} \in L_{0},\left\|l_{0}\right\|_{\Delta}>0$, что $l_{0}\left(x_{1}\right)=l_{0}\left(x_{2}\right)=\cdots=l_{0}\left(x_{n}\right)=0$, где $a=x_{1}<x_{2}<\cdots<x_{n-1}<x_{n}=b$. Поскольку $\left(p, L_{0}\right)$ - система единственности на $C(\Delta)$, а точки $a$ и $b$ принадлежат носителю $M(p, \Delta), N(p, \Delta)=\varnothing$, то по лемме 4 для каждой однозначной на $X=\{a, b\}$ ветви $\sigma(x)$ сигнатуры веса $p$ найдется полином $l \in L_{0}$ такой, что $\operatorname{sign} l(x)=-\sigma(x)$ $\forall x \in X$. Если $n:=\operatorname{dim} L_{0}-$ нечетное число, то возьмем полином $l(x)$, принимающий в точках $a$ и $b$ значения противоположного знака, если $n-$ четное число, то одного знака (это всегда можно сделать в силу условия $a \vee b \in F(p, \Delta)$ ). Нетрудно увидеть, что и в том и в другом случае полином $l+t l_{0}$ при некотором (достаточно большом по модулю) $t \in \mathbb{R}$ имеет на $(a, b)$ не менее $n$ нулей, что невозможно, так как подпространство $L_{0}$ - чебьшёвское на $(a, b)$. Теорема доказана.

ТЕОРема 11. Для того чтобъ вес $p \in \mathscr{P}(\Delta)$ бил обобщенно-хааровским на отрезке $\Delta=[a, b]$ необходимо и достаточно, чтобы одновременно выполнялись следующие условия:

1) $H(p, \Delta)=(a, b)$;

2) $N(p, \Delta) \subset(a, b)$;

3) $a \vee b \in F(p, \Delta)$.

ДокаЗАТЕЛЬство. Необходимость. 1) Пусть $H(p, \Delta) \neq(a, b)$. Тогда по второй части теоремы $8 \mathscr{Z}^{*}(p, E) \neq \mathscr{Z} T(E)$.

2) Пусть $a \vee b \in N(p, \Delta)$. Возьмем подпространство $L \in \mathscr{Z}(\Delta)$ такое, что $L-$ чебьшёвское на каждом из промежутков $[a, b)$ и $(a, b]$, но не чебышёвское на $[a, b]$ (в остальном произвольное). Тогда пара $(p, L)$ принадлежит множеству $\mathscr{Z} *(p, \Delta)$ (см. леммы 2 и 8), но не принадлежит $\mathscr{Z} T(\Delta)$. Противоречие.

3) См. соответствующий пункт доказательства необходимости в теореме 10 (в нем следует лишш $\mathscr{Z}(p, \Delta)$ заменить на $\mathscr{Z} *(p, \Delta))$.

Достаточность. Поскольку $H(p, \Delta)=(a, b), N(p, \Delta) \subset(a, b)$, то по следствию 5 если подпространство $L$ принадлежит $\mathscr{Z} *(p, \Delta)$, то $L$ является чебышёвским на промежутках $[a, b)$ и $(a, b]$. Далее доказательство проходит так же, как в п. 2) доказательства достаточности теоремы 10 (с заменой $\mathscr{Z}(p, \Delta)$ на $\left.\mathscr{Z}^{*}(p, \Delta)\right)$. Теорема доказана.

Возвращаясь к исходной задаче описания всех пар $(p, L)$, являющихся системами единственности, отметим, что теоремы этого параграфа, выделяя определенньй аспект проблемы, показывают достаточную эффективность установленного в 
$\S 1$ общего критерия единственности (теорема 2). Еще один аспект проблемы выделен в следуюшей нерешенной задаче: дать описание всех конечномерных подпространств $L$ в $C(E), E$ - компакт, для каждого из которых существует вес $p \in \mathscr{P}(E)$ с пустым открытым ядром своего аннулятора $N(p, E)$ (см. замечание 8) такой, что пара $(p, L)$ является системой единственности на $C(E)$.

\section{Список литературы}

1. Borel E. Lecons sur les fonctions de variables réelles. Paris: Gauthier-Villars, 1905.

2. Ахиезер Н. И. Лекции по теории аппроксимации. М.: Физматгиз, 1965.

3. Haar A. Die Minkowskische Geometrie und die Annäherung an stetige Funktionen // Math. Ann. 1918. V. 78. P. 293-311.

4. Лоран П.-ЖК. Аптроксимация и оптимизация. М.: Мир, 1975.

5. Крейн М. Г., Нудельман А. А. Проблема моментов Маркова и экстремальные задачи. М.: Наука, 1973.

6. Долженко Е.П., Севастьянов Е.А. Знакочувствительные аптроксимации. Пространство знакочувствительных весов. Жесткость и свобода системы // Докл. АН. 1993. T. 332. №6. C. $686-689$.

7. Галеев Э. М., Тихомиров В. М. Краткий курс теории экстремальнтх задач. М.: Изд-во МГУ, 1989.

8. Колмогоров А. Н., Фомин С. В. Элементы теории функций и функционального анализа. М.: Наука, 1972.

9. Бәр Р. Теория разрьвных функций. М.-Л.: Гостехиздат, 1932.

10. Бернштейн $C ., H$. Экстремальные свойства полиномов и наилучшее приближение непрерывных функций одной вещественной переменной. М.-Л.: ОНТИ, 1937.

11. Даугавет И. К. Введение в теорию приближения функций. Л.: Изд-во ЛГУ, 1977.

12. Тихомиров В. М. Некоторые вопросы теории приближений. М.: Изд-во МГУ, 1976.

13. Колмогоров А. Н. Замечание по поводу многочленов П. Л. Чебышёва, наименее уклоняющихся от заданной функции // УМН. 1948. Т. 3. №1. С. 216-221.

14. Куратовский К. Топология. М.: Мир, 1966.

15. Baire R. Thése Ph. Sur les fonctions de variables réelles // Ann. De Math. TURA ed. Appl. Sér. 3. V. 3, 1899.

16. Окстоби Дж. Мера и категория. М.: Мир, 1974.

17. Дзядык В. К. Введение в теорию равномерного приближения функций полиномами. М.: Наука, 1977.

18. Рокафеллар Р. Вьпуклый анализ. М.: Мир, 1973.

Московский институт

Поступила в редакцию

коммунального хозяйства и строительства

13.09 .1995 